\title{
BLOCK BOOTSTRAP THEORY FOR MULTIVARIATE INTEGRATED AND COINTEGRATED PROCESSES
}

\author{
CARSTEN JENTSCH, EFSTATHIOS PAPARODITIS, AND DIMITRIS N. POLITIS
}

\begin{abstract}
We develop some asymptotic theory for applications of block bootstrap resampling schemes to multivariate integrated and cointegrated time series. It is proved that a multivariate, continuous-path block bootstrap scheme applied to a full rank integrated process, succeeds in estimating consistently the distribution of the least squares estimators in both, the regression and the spurious regression case. Furthermore, it is shown that the same block resampling scheme does not succeed in estimating the distribution of the parameter estimators in the case of cointegrated time series. For this situation, a modified block resampling scheme, the so-called residual based block bootstrap, is investigated and its validity for approximating the distribution of the regression parameters is established. The performance of the proposed block bootstrap procedures is illustrated in a short simulation study.
\end{abstract}

JEL classification: C15; C32

\section{INTRODUCTION}

The block bootstrap methodology, Künsch (1989), Liu and Singh (1992), is a general resampling scheme applicable to time series data obeying a weak dependence structure; see Lahiri (2003) for an overview. Since then, block bootstrap has been successfully applied to a wide range of inference problems in statistics and econometrics. In particular, it has become a useful tool for various estimation and testing problems extensively studied in econometrics literature. In the univariate case, applications of block resampling schemes to integrated processes have been considered by Paparoditis and Politis (2003), Parker, Paparoditis and Politis (2006) and Phillips (2010). For the multivariate case, Li and Maddala (1997) and Badillo, Belaire-Franch and Reverte (2010) investigate the usefulness of block bootstrap methods for small sample inference in cointegration regression models and for cointegration rank testing. However, despite these applied papers, in the multivariate context few is known about the theoretical properties of the block bootstrap used for statistical inference.

However, diverse other bootstrap approaches have been applied and studied in several estimation and testing contexts in multivariate integrated and cointegrated models. Chang, Park and Song (2006) propose a sieve bootstrap for estimation and testing of linear hypothesis in cointegrating regressions. They prove bootstrap consistency not only for the OLS estimator and corresponding test statistics, but also for the case of asymptotically efficient estimation and hypothesis testing. Size and power properties of bootstrap hypotheses tests on cointegrating vectors have been compared to Bartlett-corrected tests in Omtzigt and Fachin (2006), where it is pointed out that both types of tests must be based on the unrestricted estimates of the cointegrating vectors not to lose power. In a reduced rank VAR model, Swensen $(2006,2009)$ applied a residual-based bootstrap procedure for testing and determining the cointegration rank. He proves bootstrap consistency for the likelihood ratio test statistic (trace statistic) which consequently leads to a valid sequential procedure to determine the cointegration rank of the system.

\footnotetext{
Date: July 10, 2014.

Key words and phrases. Block bootstrap, bootstrap consistency, spurious regression, functional limit theorem, continuous-path block bootstrap, model-based block bootstrap.
} 
Since this influential paper, this bootstrap testing methodology has been refined in several directions. In Trenkler (2009), the potential benefit of a prior adjustment for deterministic terms before bootstrapping is investigated and consistency is proved for the adjusted system cointegration tests. Cavaliere, Rahbek and Taylor $(2010 \mathrm{a}, \mathrm{b})$ propose to use wild bootstrap to extend the methodology of Swensen (2006) to cases where conditional and unconditional heteroskedasticity is present in the innovations. Swensen (2011) considers reduced rank autoregressive models that include also stationary regressors and provides theory. Cavaliere, Rahbek and Taylor (2012) proposed to execute the bootstrap procedure with restricted parameter estimates, which allows for bootstrap consistency under weaker conditions than used by Swensen (2006). Its generalization to heteroskedastic innovations has been addressed in Cavaliere, Rahbek and Taylor (2014).

The aim of this paper is to complement the existing literature by filling the gap of missing theory for the block bootstrap and to provide some asymptotic theory that justifies applications of block bootstrap methods to multivariate integrated and/or cointegrated processes. In particular, we establish asymptotic theory not only under linearity, but also for innovations fulfilling some suitable strong mixing conditions. We first consider a so-called, full rank, multivariate integrated process and investigate properties of a block bootstrap resampling scheme that is based on block bootstrapping of the centered differences of the observed multivariate time series. This resampling scheme is a multivariate version of the so-called Continuous-Path Block bootstrap (CBB), proposed in the univariate context by Paparoditis and Politis (2001). Based on CBBgenerated multivariate bootstrap observations, we first establish a functional limit theorem for the bootstrap partial sum process. Using this basic result, we then prove asymptotic validity of the $\mathrm{CBB}$ method applied to the matrix of the least squares estimators in the full rank integrated case. We also show that by means of the same bootstrap procedure, the distribution of the least squares estimators for the spurious regression as coined by Phillips (1986) can be also successfully approximated. This complements the results in Phillips (2001), who investigated the applicability of several bootstrap approaches in this framework. Further, we show that the same bootstrap method based on resampling blocks of the (centered) differences does not work, however, in the case of multivariate cointegrated processes. This is due to the fact that in this case the block resampling scheme based on differences does not mimic correctly the cointegration structure of the underlying process. For this kind of processes a Residual-based Block Bootstrap (RBB) is more appropriate. This procedure is based on block bootstrapping of the (centered) residuals of a regression fit obtained using the cointegration relations. We prove that the RBB is asymptotically valid in approximating the distribution of the least squares estimators of the cointegration matrix.

The paper is organized as follows. Section 2 describes the CBB procedure and establishes a basic functional limit theorem for the block bootstrap generated partial sum process. Applications of the CBB bootstrap scheme to multivariate integrated processes are then considered and asymptotic validity for the full rank integrated case and in the spurious regression case are established. Section 3 deals with cointegrated multivariate processes. It is first demonstrated that the CBB applied to the least squares estimator fails. A residual based block bootstrap (RBB) resampling scheme is then discussed. It is shown, that for cointegrated processes the RBB resampling scheme leads to an asymptotically valid approximation of the distribution of the least squares cointegration matrix. Section 4 summarizes our findings while all proofs are deferred to Section 5.

\section{Block Bootstrap for Integrated Processes}

\subsection{Preliminaries.}


Suppose we have $m$-variate time series data $X_{1}, \ldots, X_{n}$ plus one additional pre-sample value $X_{0}$ at hand stemming from a stochastic process $\left\{X_{t}, t \in \mathbb{N}_{0}\right\}$ where the $X_{t}$ 's are $\mathbb{R}^{m}$-valued random variables. Throughout this paper, we will also denote by $X_{1 t}$ the $m_{1}$-dimensional vector that contains the first $m_{1}$ coordinates of $X_{t}$ and by $X_{2 t}$ the $m_{2}$-dimensional vector that contains the last $m_{2}=m-m_{1}$ coordinates of $X_{t}$ for some $0 \leq m_{1} \leq m$. In this section, we assume that $\left\{X_{t}, t \in \mathbb{N}_{0}\right\}$ is a so-called, $m$-dimensional, full-rank random walk, i.e., $X_{t}$ follows the model

$$
X_{t}=X_{t-1}+U_{t}, \quad t \in \mathbb{N},
$$

where $X_{0}=O_{a s}(1)$ is random following a certain fixed distribution and $\left\{U_{t}, t \in \mathbb{N}\right\}$ is a stationary process satisfying either Assumption 2.1 or Assumption 2.2 below. By these assumptions, $\left\{U_{t}, t \in \mathbb{N}\right\}$ is allowed to be either a linear process or a process fulfilling some general strong mixing conditions; see also Phillips (1988) and Paparoditis and Politis (2003).

\section{Assumption 2.1.}

The process $\left\{U_{t}, t \in \mathbb{N}\right\}$ is a sequence of $m$-variate random variables satisfying

$$
U_{t}=\sum_{\nu=0}^{\infty} \psi_{\nu} \epsilon_{t-\nu}
$$

where $\psi_{\nu}=\left(\psi_{\nu, i j}\right)_{i, j=1, \ldots, m}, \sum_{\nu=1}^{\infty} \nu\left|\psi_{\nu, i j}\right|<\infty$ for each $i, j=1, \ldots, m,\left(\epsilon_{t}, t \in \mathbb{Z}\right)$ is an $m$ variate sequence of i.i.d. random variables with $E\left(\epsilon_{t}\right)=0, E\left(\epsilon_{i t}^{4}\right)<\infty$ for $i=1,2, \ldots, m$ and $E\left(\epsilon_{t} \epsilon_{t}^{\prime}\right)=\Sigma>0$. Furthermore,

$$
\Omega=2 \pi f(0)=\left(\sum_{\nu=0}^{\infty} \psi_{\nu}\right) \Sigma\left(\sum_{\nu=0}^{\infty} \psi_{\nu}\right)^{\prime}>0,
$$

where $f(\omega)$ denotes the spectral density matrix of $\left\{U_{t}, t \in \mathbb{N}\right\}$.

\section{Assumption 2.2.}

The process $\left\{U_{t}, t \in \mathbb{N}\right\}$ is a sequence of strictly stationary, strong mixing $m$-variate random variables satisfying $E\left(U_{t}\right)=0, E\left|U_{i 1}\right|^{\beta+\epsilon}<\infty, i=1, \ldots, m$ for some $\beta>2, \epsilon>0$ and $\sum_{k=1}^{\infty} \alpha(k)^{1-2 / \beta}<\infty$. The $\alpha$-mixing coefficient $\alpha(k)$ is defined by

$$
\alpha(k)=\sup _{A \in \mathcal{F}_{t+k}^{\infty}, B \in \mathcal{F}_{-\infty}^{t}}|P(A \cap B)-P(A) P(B)|,
$$

where $\mathcal{F}_{t+k}^{\infty}=\sigma\left(X_{t+k}, X_{t+k+1}, \ldots\right), \mathcal{F}_{-\infty}^{t}=\sigma\left(X_{t}, X_{t-1}, \ldots\right)$ and $\sigma(Y)$ denotes the $\sigma$-algebra generated by the random variable $Y$. Furthermore, it holds true that $E\left(n^{-1} S_{n} S_{n}^{\prime}\right) \rightarrow \Omega>0$ as $n \rightarrow \infty$, where $S_{t}=\sum_{j=1}^{t} U_{j}$.

Some remarks on the two sets of assumptions are in order. Assumption 2.1 imposes linearity of the process $\left\{U_{t}\right\}$ with sufficiently fast decaying coefficient matrix entries. In contrast, $\left\{U_{t}\right\}$ is assumed to fulfill a suitable strong mixing condition in Assumption 2.2 that is just strong enough to prove limit theorems. In both conditions, the long-run variance $\Omega$ of $\left\{U_{t}\right\}$ is assumed to be non-singular to ensure the existence of its inverse.

The following notation borrowed e.g. from Phillips (1988) is used in the sequel. We set

$$
\Omega=\Gamma(0)+\sum_{h=1}^{\infty} \Gamma(h)+\sum_{h=1}^{\infty} \Gamma(-h)=\Omega_{0}+\Omega_{1}+\Omega_{1}^{\prime},
$$

where $\Gamma(h)=\operatorname{Cov}\left(U_{t+h}, U_{t}\right)=E\left(U_{t+h} U_{t}^{\prime}\right)$ denotes the covariance matrix at lag $h \in \mathbb{Z}$ of the innovation process $\left\{U_{t}\right\}$. 


\subsection{The Continuous-path Block Bootstrap (CBB).}

We first describe a multivariate version of the so-called Continuous-path Block Bootstrap (CBB) as proposed by Paparoditis and Politis (2003). This general block bootstrap resampling scheme will be applied in the sequel in order to approximate the distribution of statistics calculated using time series data $X_{0}, X_{1}, \ldots, X_{n}$ stemming from the process (2.1), where $\left\{U_{t}\right\}$ satisfies Assumption 2.1 or Assumption 2.2. More precisely, the multivariate CBB consists of the following four steps.

Step 1. Compute the differences $U_{t}=\Delta X_{t}=X_{t}-X_{t-1}, t=1, \ldots, n$.

Step 2. Choose a block length $b<n$ and let $k$ be the smallest number of blocks needed to get a bootstrap sample of length $l=k b$, such that $l \geq n$. Let $i_{0}, \ldots, i_{k-1}$ be i.i.d. random variables uniformly distributed on the set $\{0,1,2, \ldots, n-b\}$.

Step 3. Let $U_{1}^{*}, \ldots, U_{l}^{*}$ be a moving block bootstrap sample, where for $j=1,2, \ldots, b$ and $m=$ $0,1,2, \ldots, k-1$

$$
U_{m b+j}^{*}=\widehat{U}_{i_{m}+j}:=U_{i_{m}+j}-E^{*}\left(U_{i_{m}+j}\right)=U_{i_{m}+j}-\frac{1}{n-b+1} \sum_{\tau=0}^{n-b} U_{\tau+j} .
$$

Step 4. Generate then the bootstrap pseudo-time series $X_{1}^{*}, \ldots, X_{l}^{*}$, as

$$
X_{t}^{*}= \begin{cases}X_{0}, & t=0 \\ X_{t-1}^{*}+U_{t}^{*}, & t \in\{1, \ldots, l\} .\end{cases}
$$

Remark 2.1. Note that the centering of the bootstrap sample in (2.3) is tailor-made for the moving block bootstrap and adjusted centering has to be applied for other approaches as e.g. non-overlapping block bootstrap, cyclical block bootstrap or stationary bootstrap.

\subsection{A Functional Limit Theorem (FLT) for the bootstrap partial sum process.}

We first establish a basic result which is useful to prove consistency of the CBB resampling scheme. In fact, the asymptotic theory for establishing bootstrap consistency for statistics based on time series $X_{1}, X_{2}, \ldots, X_{n}$ relies to a large extent on the asymptotic behavior of the standardized partial sum process $\left\{S_{l}^{*}(\nu), 0 \leq \nu \leq 1\right\}$, which is defined by

$$
\begin{aligned}
& S_{l}^{*}(\nu)=\frac{1}{\sqrt{l}}\left(\Omega_{l}^{*}\right)^{-1 / 2} \sum_{t=1}^{j-1} U_{t}^{*}, \quad \text { for } \quad \frac{j-1}{l} \leq \nu<\frac{j}{l}, \quad j=1, \ldots, l, \\
& S_{l}^{*}(\nu)=\frac{1}{\sqrt{l}}\left(\Omega_{l}^{*}\right)^{-1 / 2} \sum_{t=1}^{l} U_{t}^{*}, \quad \text { for } \quad \nu=1 .
\end{aligned}
$$

By convention, summations over empty sets are zero and $\left(\Omega_{l}^{*}\right)^{1 / 2}$ is the symmetric (positive semi-definite) square root of

$$
\Omega_{l}^{*}=\operatorname{Var}^{*}\left(\frac{1}{\sqrt{l}} \sum_{t=1}^{l} U_{t}^{*}\right)=\frac{1}{b} \sum_{s_{1}, s_{2}=1}^{b} \frac{1}{n-b+1} \sum_{t=0}^{n-b} \widehat{U}_{t+s_{1}} \widehat{U}_{t+s_{2}}^{\prime}, .
$$

Notice that by Assumption 2.1 or Assumption 2.2, $\Omega_{l}^{*}$ is positive definite with probability tending to one.

Observe that $S_{l}^{*}(\cdot)$ is a random element taking values in the space $D[0,1]^{m}=D[0,1] \times \cdots \times$ $D[0,1]$, the product metric space of all real valued vector functions on $[0,1]$ that are right continuous at each element of $[0,1]$ and possess finite left limits. Each component space $D[0,1]$ is endowed with the Skorohod metric, denoted by $d$, which ensures separability of the metric 
space. For the product space $D[0,1]^{m}$, the metric $\tilde{d}$ is defined by

$$
\tilde{d}(x, y)=\max _{i \in\{1,2, \ldots, m\}}\left\{d\left(x_{i}, y_{i}\right): x_{i}, y_{i} \in D[0,1]\right\} .
$$

In the following theorem, we prove that conditional on the sample $X_{1}, \ldots, X_{n}$, the standardized CBB bootstrap partial sum process (2.5), converges weakly to an $m$-dimensional standard Wiener process, where each element of $W(t)$ is a univariate Wiener process and the elements of $W(t)$ are independent from each other. The notation $S_{l}^{*} \Rightarrow W$ in probability, means that the distance between the law of $S_{l}^{*}=S_{l}^{*}(\cdot)$ and the law of $W$ tends to zero in probability for any distance metrizing weak convergence.

Theorem 2.1. Let $\left\{X_{t}, t \in \mathbb{N}_{0}\right\}$ be an $m$-dimensional stochastic process following (2.1) and assume that the process $\left\{U_{t}, t \in \mathbb{N}\right\}$ satisfies Assumption 2.1 or Assumption 2.2. If $b \rightarrow \infty$ as $n \rightarrow \infty$ such that $b / \sqrt{n} \rightarrow 0$, then

$$
S_{l}^{*} \Rightarrow W
$$

in probability, respectively, where $\left\{W(t)=\left(W_{1}(t), \ldots, W_{m}(t)\right)^{\prime}, t \in[0,1]\right\}$ is here and throughout this paper an m-dimensional standard Wiener process on $[0,1]$, i.e. each element $W_{i}(\cdot)$ is a univariate Wiener process and the elements of $W(\cdot)$ are independent.

We next discuss some cases where Theorem 2.1 is employed to establish asymptotic properties of block-bootstrap based, statistical inference procedures.

\subsection{Applications to Regression Estimators.}

\subsubsection{Regressing $X_{t}$ on $X_{t-1}$.}

Consider the least squares (LS) estimator $\widehat{A}$ obtained by regressing $X_{t}$ on $X_{t-1}$, that is,

$$
\widehat{A}=\left(\sum_{t=2}^{n} X_{t} X_{t-1}^{\prime}\right)\left(\sum_{t=2}^{n} X_{t-1} X_{t-1}^{\prime}\right)^{-1} .
$$

We want to approximate the unknown distribution of $n\left(\widehat{A}-I_{m}\right)$ by the bootstrap distribution of $l\left(\widehat{A}^{*}-I_{m}\right)$, where

$$
\widehat{A}^{*}=\left(\sum_{t=2}^{l} X_{t}^{*} X_{t-1}^{*^{\prime}}\right)\left(\sum_{t=2}^{l} X_{t-1}^{*} X_{t-1}^{*^{\prime}}\right)^{-1},
$$

$I_{m}$ is the $(m \times m)$ unity matrix and $X_{1}^{*}, \ldots, X_{l}^{*}$ is generated using the CBB scheme described in Section 2.2. As

$$
n\left(\widehat{A}-I_{m}\right) \Rightarrow\left\{\Omega^{1 / 2} \int_{0}^{1} W(t) d W(t)^{\prime} \Omega^{1 / 2}+\Omega_{1}\right\}\left\{\Omega^{1 / 2} \int_{0}^{1} W(t) W(t)^{\prime} d t \Omega^{1 / 2}\right\}^{-1}
$$

holds under Assumptions 2.1 [cf. Lütkepohl (2006), Proposition C.18] or 2.2 [cf. Phillips and Durlauf (1986), Theorem 2.1], the following theorem establishes validity of the multivariate CBB procedure for approximating the distribution of the least squares estimator $\widehat{A}$.

Theorem 2.2. Under the assumptions of Theorem 2.1, conditionally on $X_{1}, X_{2}, \ldots, X_{n}$, it holds true that

$$
l\left(\widehat{A}^{*}-I_{m}\right) \Rightarrow\left\{\Omega^{1 / 2} \int_{0}^{1} W(t) d W(t)^{\prime} \Omega^{1 / 2}+\Omega_{1}\right\}\left\{\Omega^{1 / 2} \int_{0}^{1} W(t) W(t)^{\prime} d t \Omega^{1 / 2}\right\}^{-1}
$$

in probability, where $\Omega$ and $\Omega_{1}$ are defined in (2.2). 
2.4.2. Spurious Regression of $X_{1 t}$ on $X_{2 t}$ for $m_{1}=1$.

Consider next the so-called spurious regression case, where we regress the first coordinate of $X_{t}$ on the remaining ones, while the underlying process is the full rank random walk (2.1). By using the notation introduced in Section 2.1, this means, we regress $X_{1 t}$ on $X_{2 t}$ with $m_{1}=1$. Let $\widehat{\alpha}$ and $\widehat{\beta}$ be the least squares estimators of $\alpha$ and $\beta$ when one fits the (spurious regression) model

$$
X_{1 t}=\alpha+\beta^{\prime} X_{2 t}+\epsilon_{t},
$$

to the time series at hand. More specifically,

$$
\widehat{\beta}=\left\{\frac{1}{n^{2}} \sum_{t=1}^{n}\left(X_{2 t}-\bar{X}_{2}\right)\left(X_{2 t}-\bar{X}_{2}\right)^{\prime}\right\}^{-1}\left\{\frac{1}{n^{2}} \sum_{t=1}^{n}\left(X_{2 t}-\bar{X}_{2}\right)\left(X_{1 t}-\bar{X}_{1}\right)\right\}
$$

and $\widehat{\alpha}=\bar{X}_{1}-\bar{X}_{2}^{\prime} \widehat{\beta}$, where $\bar{X}_{1}=\frac{1}{n} \sum_{t=1}^{n} X_{1 t}$ and $\bar{X}_{2}=\frac{1}{n} \sum_{t=1}^{n} X_{2 t}$. From Phillips (1986), Theorem 2 and under Hamilton (1994), Proposition 18.2, we get under Assumptions 2.2 or 2.1, respectively, that

$$
\begin{aligned}
\frac{1}{\sqrt{n}} \widehat{\alpha} & \Rightarrow b_{1}-b_{2}^{\prime} A_{22}^{-1} a_{21} \\
\widehat{\beta} & \Rightarrow A_{22}^{-1} a_{21},
\end{aligned}
$$

where $a_{11}, b_{1}, a_{12}, a_{21}, b_{2}$ and $A_{22}$ are random variables such that $a_{11}, b_{1}$ take values in $\mathbb{R}$, $a_{12}, a_{21}, b_{2}$ in $\mathbb{R}^{m-1}$ and $A_{22}$ in $\mathbb{R}^{(m-1) \times(m-1)}$ and

$$
\begin{aligned}
A & =\left(\begin{array}{ll}
a_{11} & a_{12}^{\prime} \\
a_{21} & A_{22}
\end{array}\right)=\Omega^{1 / 2}\left\{\int_{0}^{1} W(t) W^{\prime}(t) d t-\int_{0}^{1} W(t) d t \int_{0}^{1} W^{\prime}(t) d t\right\} \Omega^{1 / 2}, \\
b & =\left(\begin{array}{l}
b_{1} \\
b_{2}
\end{array}\right)=\Omega^{1 / 2} \int_{0}^{1} W(t) d t .
\end{aligned}
$$

Further, let $\widehat{\alpha}^{*}$ and $\widehat{\beta}^{*}$ be the bootstrap analogues of $\widehat{\alpha}$ and of $\widehat{\beta}$, i.e., the least squares estimators of $\alpha$ and $\beta$ in running the regression

$$
X_{1 t}^{*}=\alpha+\beta^{\prime} X_{2 t}^{*}+\epsilon_{t}
$$

using the bootstrap pseudo-time series $X_{1}^{*}, \ldots, X_{l}^{*}$ generated by the CBB scheme of Section 2.2. Here, $\bar{X}_{1}^{*}=\frac{1}{l} \sum_{t=1}^{l} X_{1 t}^{*}$ and $\bar{X}_{2}^{*}=\frac{1}{l} \sum_{t=1}^{l} X_{2 t}^{*}$. The corresponding bootstrap estimator is then given by

$$
\widehat{\beta}^{*}=\left\{\frac{1}{l^{2}} \sum_{t=1}^{l}\left(X_{2 t}^{*}-\bar{X}_{2}^{*}\right)\left(X_{2 t}^{*}-\bar{X}_{2}^{*}\right)^{\prime}\right\}^{-1}\left\{\frac{1}{l^{2}} \sum_{t=1}^{l}\left(X_{2 t}^{*}-\bar{X}_{2}^{*}\right)\left(X_{1 t}^{*}-\bar{X}_{1}^{*}\right)\right\}
$$

and $\widehat{\alpha}^{*}=\bar{X}_{1}^{*}-\bar{Z}^{* \prime} \widehat{\beta}^{*}$. We then have the following theorem.

Theorem 2.3. Under the assumptions of Theorem 2.1, it holds

$$
\begin{aligned}
\frac{1}{\sqrt{l}} \widehat{\alpha}^{*} & \Rightarrow b_{1}-b_{2}^{\prime} A_{22}^{-1} a_{21} \\
\widehat{\beta}^{*} & \Rightarrow A_{22}^{-1} a_{21}
\end{aligned}
$$

in probability, respectively.

As the above theorem in comparison to (2.11) and (2.12) shows, the CBB procedure succeeds in approximating correctly the distribution of the parameter estimators $\widehat{\alpha}$ and $\widehat{\beta}$ in the spurious regression case. 


\section{Block Bootstrap for Cointegrated Processes}

\subsection{CBB applied to cointegrated processes.}

Consider now the case where the underlying process fulfills a cointegrated relation, that is, the multivariate time series $X_{1}, \ldots, X_{n}$ is generated by the $m=m_{1}+m_{2}$-dimensional model

$$
\begin{aligned}
& X_{1 t}=B X_{2 t}+U_{1 t}, \\
& X_{2 t}=X_{2, t-1}+U_{2 t},
\end{aligned}
$$

where $X_{0}=O_{P}(1)$ is random following a certain fixed distribution, $X_{1 t}, U_{1 t}$ are $m_{1}$-dimensional, $X_{2 t}, U_{2 t}$ are $m_{2}$-dimensional, $B$ is an $\left(m_{1} \times m_{2}\right)$ matrix and $\left\{U_{t}=\left(U_{1 t}^{\prime}, U_{2 t}^{\prime}\right)^{\prime}, t \in \mathbb{N}\right\}$ is a stationary process satisfying Assumption 2.1 or Assumption 2.2. Notice that $\left\{X_{t}, t \in \mathbb{N}\right\}$ is cointegrated with cointegration rank $m_{1}$. Our goal is to investigate the properties of the CBB bootstrap in estimating the distribution of the LS-estimator $\widehat{B}$ of $B$, where

$$
\widehat{B}=\left(\sum_{t=1}^{n} X_{1 t} X_{2 t}^{\prime}\right)\left(\sum_{t=1}^{n} X_{2 t} X_{2 t}^{\prime}\right)^{-1} .
$$

Under the imposed conditions [cf. Lütkepohl (2006), Lemma 7.1 or Phillips and Durlauf (1986), Theorem 4.1], we have

$$
n(\widehat{B}-B) \Rightarrow\left[\Omega^{1 / 2} \int_{0}^{1} W(t) d W^{\prime}(t) \Omega^{1 / 2}+\Omega_{0}+\Omega_{1}\right]_{12}\left[\left[\Omega^{1 / 2} \int_{0}^{1} W(t) W^{\prime}(t) d t \Omega^{1 / 2}\right]_{22}\right]^{-1},
$$

where $\Omega_{1}, \Omega$ and $\Omega_{0}$ are defined in $(2.2)$ and $[C]_{12},[C]_{22}$ denote the $\left(m_{1} \times m_{2}\right)$ upper-right and the $\left(m_{2} \times m_{2}\right)$ lower-right part of an $(m \times m)$ matrix $C$ with $m=m_{1}+m_{2}$. The bootstrap analogue of $\widehat{B}$ is given by

$$
\widehat{B}^{*}=\left(\sum_{t=1}^{l} X_{1 t}^{*} X_{2 t}^{* \prime}\right)\left(\sum_{t=1}^{l} X_{2 t}^{*} X_{2 t}^{* \prime}\right)^{-1}
$$

where $X_{1}^{*}, X_{2}^{*}, \ldots, X_{l}^{*}$ is generated according to the CBB algorithm described in Section 2.2. Now, recall that the CBB is based on resampling blocks of the differenced time series $X_{t}-X_{t-1}$, $t=1,2, \ldots, n$, which leads to

$$
\begin{aligned}
X_{1 t}-X_{1, t-1} & =B U_{2 t}+\left(U_{1 t}-U_{1, t-1}\right) \\
& \equiv V_{1 t},
\end{aligned}
$$

and $V_{1 t} \neq U_{1 t}=X_{1 t}-B X_{2 t}$. Notice that $X_{2 t}-X_{2, t-1}=U_{2 t}$. Thus, the CBB uses the (centered) differences of the innovations $\left(V_{1 t}^{\prime}, U_{2 t}^{\prime}\right), t=1,2, \ldots, n$, to resample the blocks and not the innovations $\left(U_{1 t}^{\prime}, U_{2 t}^{\prime}\right)$ as in the case of a full rank integrated process. Furthermore, the resampled blocks obtained from a centered version of $\left(V_{1 t}^{\prime}, U_{2 t}^{\prime}\right), t=1,2, \ldots, n$, that is the pseudo replicates $\left(V_{1 t}^{* \prime}, U_{2 t}^{* \prime}\right), t=1,2, \ldots, l$, are integrated to obtain the bootstrap observations $X_{1}^{*}, X_{2}^{*}, \ldots, X_{l}^{*}$. From this we get by using $M_{t}=\left\lceil\frac{t}{b}\right\rceil-1, B_{t, m}=\min (b, t-m b), X_{1 t}^{*}=\sum_{j=1}^{t} V_{1 j}^{*}$, $X_{2 t}^{*}=\sum_{j=1}^{t} U_{2 j}^{*}$ and the definition of $V_{1 t}^{*}$ that

$$
\begin{aligned}
X_{1 t}^{*} & =B \sum_{m=0}^{M_{t}} \sum_{s=1}^{B_{t, m}} \widehat{U}_{2, i_{m}+s}+\sum_{m=0}^{M_{t}} \sum_{s=1}^{B_{t, m}}\left(\widehat{U}_{1, i_{m}+s}-\widehat{U}_{1, i_{m}+s-1}\right) \\
& =B X_{2 t}^{*}+\sum_{m=0}^{M_{t}-1}\left(\widehat{U}_{1, i_{m}+b}-\widehat{U}_{1, i_{m}}\right)+\left(\widehat{U}_{1, i_{M_{t}}+\left(t-M_{t} b\right)}-\widehat{U}_{1, i_{M_{t}}}\right),
\end{aligned}
$$

where $\widehat{U}_{1 t}$ and $\widehat{U}_{2 t}$ are the centered differences of the component processes $U_{1 t}$ and $U_{2 t}$, see also (2.3). Note also that the two last terms in the last right-hand side above are the increments 
between the last and the first random variable in each randomly selected block. Further, (3.5) leads to

$$
\begin{aligned}
\sum_{t=1}^{l} X_{1 t}^{*} X_{2 t}^{* \prime} & =B \sum_{t=1}^{l} X_{2 t}^{*} X_{2 t}^{* \prime}+\sum_{t=1}^{l} U_{1 t}^{*} X_{2 t}^{* \prime}+\sum_{t=1}^{l} \sum_{m=0}^{M_{t}-1}\left(\widehat{U}_{1, i_{m}+b}-\widehat{U}_{1, i_{m}}\right) X_{2 t}^{* \prime}-\sum_{t=1}^{l} \widehat{U}_{1, i_{M_{t}}} X_{2 t}^{* \prime} \\
& =B \sum_{t=1}^{l} X_{2 t}^{*} X_{2 t}^{* \prime}+\sum_{t=1}^{l} U_{1 t}^{*} X_{2 t}^{* \prime}+R_{1, l}+R_{2, l}
\end{aligned}
$$

with an obvious notation for $R_{1, l}$ and $R_{2, l}$. Notice that in obtaining the last equality above, $\sum_{t=1}^{l} U_{1, i_{M_{t}}+\left(t-M_{t} b\right)} X_{2 t}^{* \prime}=\sum_{t=1}^{l} U_{1 t}^{*} X_{2 t}^{* \prime}$ has been used. From the above and (3.4) we get that

$$
l\left(\widehat{B}^{*}-B\right)=\left(l^{-1} \sum_{t=1}^{l} U_{1 t}^{*} X_{2 t}^{* \prime}\right)\left(l^{-2} \sum_{t=1}^{l} X_{2 t}^{*} X_{2 t}^{* \prime}\right)^{-1}+\widetilde{R}_{1, l}+\widetilde{R}_{2, l},
$$

where

$$
\widetilde{R}_{1, l}=l^{-1} R_{1, l}\left(l^{-2} \sum_{t=1}^{l} X_{2 t}^{*} X_{2 t}^{* \prime}\right)^{-1} \quad \text { and } \quad \widetilde{R}_{2, l}=l^{-1} R_{2, l}\left(l^{-2} \sum_{t=1}^{l} X_{2 t}^{*} X_{2 t}^{* \prime}\right)^{-1} .
$$

Notice that the terms $R_{1, l}$ and $R_{2, l}$ are due to the fact that integrating the block resampled $V_{1, t}$ 's, the differences $\widehat{U}_{1, i_{m}+b}-\widehat{U}_{1, i_{m}}$ within each block do not cancel out. Now, comparing (3.6) with

$$
n(\widehat{B}-B)=\left(n^{-1} \sum_{t=1}^{n} U_{1 t} X_{2 t}^{\prime}\right)\left(n^{-2} \sum_{t=1}^{n} X_{2 t} X_{2 t}^{\prime}\right)^{-1},
$$

indicates that $\left(l^{-1} \sum_{t=1}^{l} U_{1 t}^{*} X_{2 t}^{* \prime}\right)\left(l^{-2} \sum_{t=1}^{l} X_{2 t}^{*} X_{2 t}^{* \prime}\right)^{-1}$ mimics in the bootstrap world the stochastic behavior of $\left(n^{-1} \sum_{t=1}^{n} U_{1 t} X_{2 t}^{\prime}\right)\left(n^{-2} \sum_{t=1}^{n} X_{2 t} X_{2 t}^{\prime}\right)^{-1}$. However,

$$
\frac{1}{l} R_{2, l}=\frac{1}{k} \sum_{m=0}^{k-1} \widehat{U}_{1, i_{m}} \frac{1}{b} \sum_{s=1}^{b} X_{2, m b+s}^{*}=O_{P}(1)
$$

since

$$
\frac{1}{\sqrt{b l}} \sum_{s=1}^{b} X_{2, m b+s}^{*}=\Omega^{*^{1 / 2}} \frac{1}{\sqrt{b}} \sum_{s=1}^{b} \Omega^{*^{-1 / 2}} \frac{1}{\sqrt{l}} \sum_{j=1}^{m b+s} U_{2 j}^{*}=\Omega^{*^{1 / 2}} \frac{1}{\sqrt{b}} \sum_{s=1}^{b} S_{l}^{*}((m b+s) / l)=O_{P}(1),
$$

see also the proof of Lemma 6.2(i), Section 6. This together with Lemma 6.1(iii), implies that $\widetilde{R}_{2, l}=O_{P}(1)$. Furthermore, since

$$
\frac{1}{l} R_{1, l}=\frac{1}{l} \sum_{m=0}^{k-2}\left(\widehat{U}_{1, i_{m}+b}-\widehat{U}_{1, i_{m}}\right) \sum_{t=(m+1) b+1}^{l} X_{2 t}^{*}
$$

and $l^{-3 / 2} \sum_{t=r}^{l} X_{2 t}^{*}=\Omega^{*^{1 / 2}} l^{-1} \sum_{t=r}^{l} S_{l}^{*}(t / l)$, see the proof of Lemma 6.2(i), we get that $l^{-1} R_{1, l}=$ $O_{P}(\sqrt{l k})$, and thus $\widetilde{R}_{1, l}$ does not vanish as $n \rightarrow \infty$. The above considerations and expression (3.6) lead to the conclusion that the stochastic behavior of $n(\widehat{B}-B)$ can not be successfully approximated by that of the CBB analogue $l\left(\widehat{B}^{*}-B\right)$. 


\subsection{Residual-based Block Bootstrap (RBB).}

Since the CBB applied to a cointegrated process fails due to the fact that the generated pseudotime series $X_{1}^{*}, X_{2}^{*}, \ldots, X_{n}^{*}$ mimics the behavior of a full rank random walk, we show in this section that a residual-based block bootstrap (RBB) scheme succeeds in approximating correctly the distribution of the least squares estimator $\widehat{B}$ under the cointegration model (3.1). Using this residual-based block bootstrap, we generate pseudo-time series data $X_{1}^{+}, X_{2}^{+}, \ldots, X_{n}^{+}$that retains the cointegration structure of the underlying process. This is achieved using the following block bootstrap algorithm. Notice that the notation $X_{t}^{+}$instead of $X_{t}^{*}$ is used in order to distinguish between the pseudo-time series generated by the CBB and by the following RBB algorithm.

Step 1. Compute $\widehat{B}$ and $\widetilde{U}_{t}=\left(\widetilde{U}_{1 t}^{\prime}, U_{2 t}^{\prime}\right)^{\prime}$ for $t=1, \ldots, n$ according to

$$
\begin{aligned}
& \widetilde{U}_{1 t}=X_{1 t}-\widehat{B} X_{2 t}, \\
& U_{2 t}=X_{2 t}-X_{2, t-1} .
\end{aligned}
$$

Step 2. Choose a block length $b<n$ and let $k$ be the smallest number of blocks needed to get a bootstrap sample of length $l=k b$, such that $l \geq n$. Let $i_{0}, \ldots, i_{k-1}$ be i.i.d. random variables uniformly distributed on the set $\{0,1,2, \ldots, n-b\}$.

Step 3. Let $U_{1}^{+}, \ldots, U_{l}^{*}$ with $U_{t}^{+}=\left(U_{1 t}^{+\prime}, U_{2 t}^{+\prime}\right)^{\prime}$ be a moving block bootstrap sample, where for $j=1,2, \ldots, b$ and $m=0,1,2, \ldots, k-1$,

$$
\begin{aligned}
\left(\begin{array}{c}
U_{1, m b+j}^{+} \\
U_{2, m b+j}^{+}
\end{array}\right) & =\left(\begin{array}{c}
\widehat{U}_{1, i_{m}+j} \\
\widehat{U}_{2, i_{m}+j}
\end{array}\right)=\left(\begin{array}{c}
\widetilde{U}_{1, i_{m}+j}-E^{+}\left(\widetilde{U}_{1, i_{m}+j}\right) \\
U_{2, i_{m}+j}-E^{+}\left(U_{2, i_{m}+j}\right)
\end{array}\right) \\
& =\left(\begin{array}{c}
\widetilde{U}_{1, i_{m}+j} \\
U_{2, i_{m}+j}
\end{array}\right)-\frac{1}{n-b+1} \sum_{\tau=0}^{n-b}\left(\begin{array}{c}
\widetilde{U}_{1, \tau+j} \\
U_{2, \tau+j}
\end{array}\right) .
\end{aligned}
$$

Step 4. Generate then the bootstrap pseudo-time series $\left(X_{1 t}^{+\prime}, X_{2 t}^{+\prime}\right)^{\prime}, t=1, \ldots, l$ from

$$
X_{2 t}^{+}= \begin{cases}X_{20}, & t=0 \\ X_{2, t-1}^{+}+U_{2 t}^{+}, & t \in\{1, \ldots, l\}\end{cases}
$$

and

$$
X_{1 t}^{+}=\widehat{B} X_{2 t}^{+}+U_{1 t}^{+}, \quad t \in\{1, \ldots, l\} .
$$

This leads to the estimator

$$
\widehat{B}^{+}=\left(\sum_{t=1}^{l} X_{1 t}^{+} X_{2 t}^{+\prime}\right)\left(\sum_{t=1}^{l} X_{2 t}^{+} X_{2 t}^{+\prime}\right)^{-1} .
$$

As (3.3) holds under Assumptions 2.1 or 2.2, the following theorem shows that the RBB procedure described above succeeds in mimicking correctly the random behavior of the LS-estimator $\widehat{B}$ in the cointegrated case $(3.1)$.

Theorem 3.1. Let $\left\{X_{t}, t \in \mathbb{N}_{0}\right\}$ be an $m$-dimensional stochastic process following (3.1) and assume that the process $\left\{U_{t}, t \in \mathbb{N}\right\}$ satisfies Assumption 2.1 or Assumption 2.2. If $b \rightarrow \infty$ as $n \rightarrow \infty$ such that $b / \sqrt{n} \rightarrow 0$ then

$$
l\left(\widehat{B}^{+}-\widehat{B}\right) \Rightarrow\left[\Omega^{1 / 2} \int_{0}^{1} W(t) d W^{\prime}(t) \Omega^{1 / 2}+\Omega_{0}+\Omega_{1}\right]_{12}\left[\left[\Omega^{1 / 2} \int_{0}^{1} W(t) W^{\prime}(t) d t \Omega^{1 / 2}\right]_{22}\right]^{-1}
$$

in probability, where $\Omega_{1}, \Omega$ and $\Omega_{0}$ are defined in (2.2). Here, $[C]_{12}$ and $[C]_{22}$ denote the $\left(m_{1} \times\right.$ $\left.m_{2}\right)$ upper-right and the $\left(m_{2} \times m_{2}\right)$ lower-right part of an $(m \times m)$ matrix $C$ with $m=m_{1}+m_{2}$. 

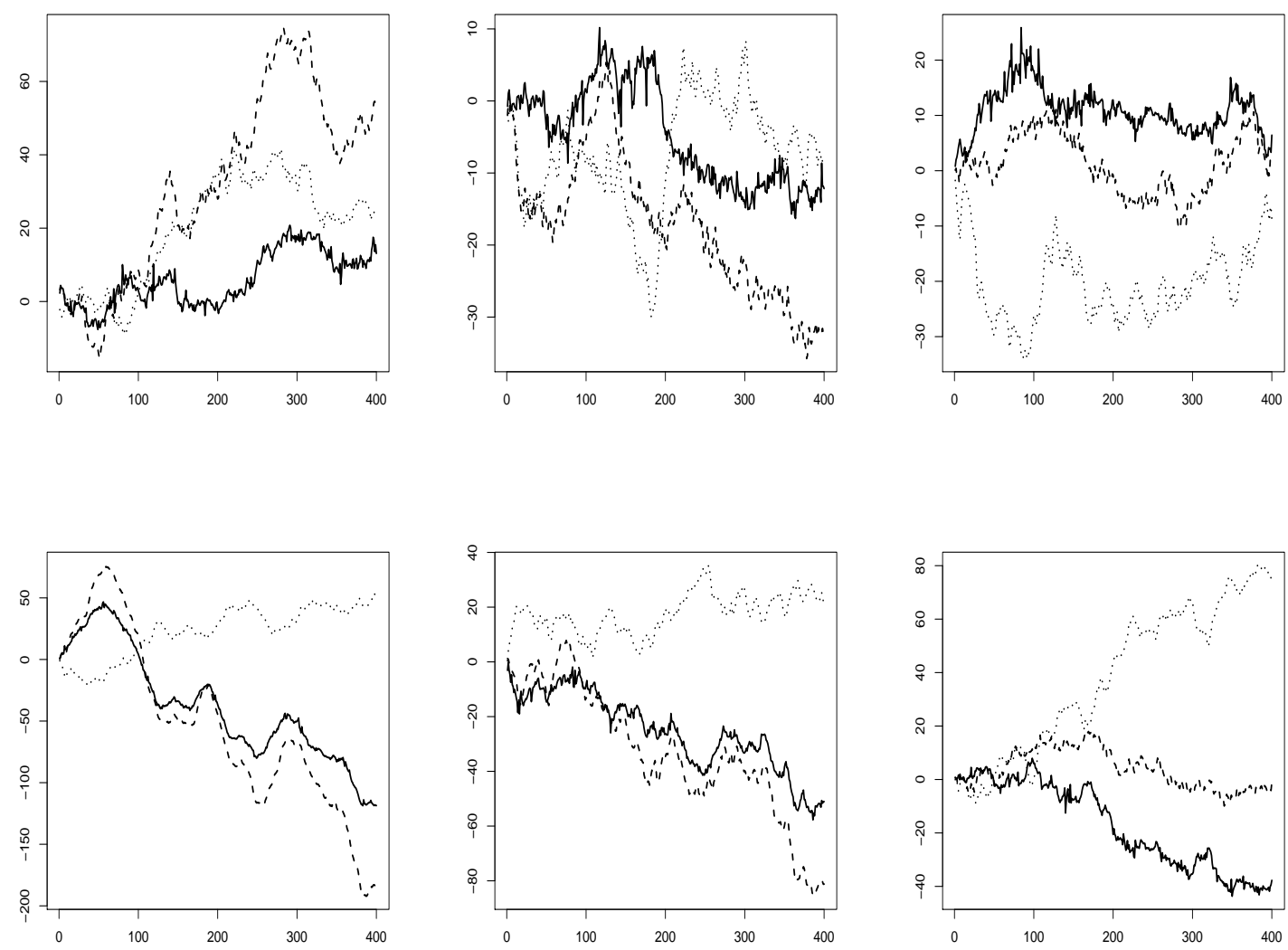

Figure 1. Realizations of the cointegrated system $\left\{X_{t}=\left(X_{1 t}, X_{2 t}^{\prime}\right)^{\prime}\right\}$ in (4.1) and (4.2) for a) MA(1) noise (upper panels) and b) AR(1) noise (lower panels) for sample size $n=400$ and $\delta \in\{0.9,0.5,0.1\}$ (from left to right). $\left\{X_{1 t}\right\}$ (solid) and both components of $\left\{X_{2 t}\right\}$ (dashed and dotted) are shown.

\section{Simulations}

In this section, we illustrate the bootstrap performance of the CBB and the RBB as proposed in the previous sections by means of coverage rates of confidence intervals and by estimated standard deviations. To cover jointly estimates for (purely) integrated as well as cointegrated multivariate time series processes, we consider data $X_{1}, \ldots, X_{n}$ from the trivariate cointegrated system

$$
\begin{aligned}
& X_{1 t}=B X_{2 t}+U_{1 t}, \\
& X_{2 t}=X_{2, t-1}+U_{2 t},
\end{aligned}
$$

where we set $X_{0}=0$. In this setup, $X_{1 t}, U_{1 t}$ are 1-dimensional, $X_{2 t}, U_{2 t}$ are 2-dimensional, $B$ is a $(1 \times 2)$ cointegration matrix and $\left\{U_{t}=\left(U_{1 t}, U_{2 t}^{\prime}\right)^{\prime}, t \in \mathbb{N}\right\}$ is a 3 -dimensional stationary process. In this section, we specify the latter process as follows. Let $U_{1 t}=e_{t} e_{t-1}$, where $\left\{e_{t}, t \in \mathbb{N}_{0}\right\}$ is i.i.d. and follows a standard normal distribution $e_{t} \sim \mathcal{N}(0,1)$. The bivariate process $\left\{U_{2 t}, t \in \mathbb{N}\right\}$ follows either an

a) $\operatorname{VMA}(1)$ model $U_{2 t}=\Psi \epsilon_{t-1}+\epsilon_{t}$

or an 


\begin{tabular}{|c|c|c|c|c|c|c|c|c|c|c|c|c|c|}
\hline \multicolumn{2}{|c|}{$\mathrm{MA}(1), \delta=0.9$} & \multicolumn{4}{|c|}{$\begin{array}{l}n=100 \\
n\end{array}$} & \multicolumn{4}{|c|}{$n=400$} & \multicolumn{4}{|c|}{$\bar{n} n=1200$} \\
\hline 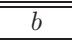 & & $\bar{\sigma} \sigma$ & mean $\left(\widehat{\sigma}^{*}\right)$ & 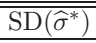 & $\operatorname{MSE}\left(\widehat{\sigma}^{*}\right)$ & $\bar{\sigma}$ & mean $\left(\widehat{\sigma}^{*}\right)$ & $\overline{\mathrm{SD}\left(\widehat{\sigma}^{*}\right)}$ & $\operatorname{MSE}\left(\widehat{\sigma}^{*}\right)$ & $\overline{\bar{\sigma}}$ & mean $\left(\widehat{\sigma}^{*}\right)$ & $\overline{\overline{\mathrm{SD}}\left(\widehat{\sigma}^{*}\right)}$ & $\operatorname{MSE}\left(\widehat{\sigma}^{*}\right)$ \\
\hline \multirow[t]{4}{*}{$\bar{c} n^{1 / 3}$} & $\widehat{\widehat{A_{11}}}$ & 3.004 & 3.451 & (0.476 & 0.427 & 2.992 & 3.221 & 0.296 & 0.140 & 3.004 & 3.164 & 0.250 & 0.088 \\
\hline & $\widehat{A}_{12}$ & 4.291 & 4.647 & 0.788 & 0.748 & 4.292 & 4.460 & 0.521 & 0.299 & 4.294 & 4.376 & 0.374 & 0.147 \\
\hline & $\widehat{A}_{21}$ & 3.224 & 3.542 & 0.609 & 0.472 & 3.197 & 3.344 & 0.366 & 0.156 & 3.230 & 3.288 & 0.275 & 0.079 \\
\hline & $\widehat{A}_{22}$ & 3.124 & 3.572 & 0.508 & 0.459 & 3.134 & 3.345 & 0.356 & 0.171 & 3.163 & 3.278 & 0.302 & 0.104 \\
\hline \multirow[t]{4}{*}{$3 n^{1 / 3}$} & $\widehat{A}_{11}$ & 3.004 & 3.586 & 0.745 & 0.894 & 2.992 & 3.302 & 0.517 & 0.363 & 3.004 & 3.182 & 0.370 & 0.168 \\
\hline & $\widehat{A}_{12}$ & 4.291 & 4.824 & 1.229 & 1.793 & 4.292 & 4.621 & 0.796 & 0.742 & 4.294 & 4.438 & 0.520 & 0.291 \\
\hline & $\widehat{A}_{21}$ & 3.224 & 3.686 & 0.909 & 1.040 & 3.197 & 3.391 & 0.552 & 0.342 & 3.230 & 3.325 & 0.385 & 0.157 \\
\hline & $\widehat{A}_{22}$ & 3.124 & 3.721 & 0.891 & 1.150 & 3.134 & 3.433 & 0.576 & 0.421 & 3.163 & 3.308 & 0.438 & 0.213 \\
\hline \multirow[t]{4}{*}{$5 n^{1 / 3}$} & $\widehat{A_{11}}$ & 3.004 & 3.595 & 0.983 & 1.316 & 2.992 & 3.423 & 0.636 & 0.591 & 3.004 & 3.279 & 0.485 & 0.311 \\
\hline & $\widehat{A}_{12}$ & 4.291 & 4.990 & 1.493 & 2.718 & 4.292 & 4.761 & 1.008 & 1.235 & 4.294 & 4.530 & 0.680 & 0.519 \\
\hline & $\widehat{A}_{21}$ & 3.224 & 3.617 & 1.068 & 1.295 & 3.197 & 3.490 & 0.728 & 0.616 & 3.230 & 3.409 & 0.482 & 0.265 \\
\hline & $\widehat{A}_{22}$ & 3.124 & 3.813 & 1.060 & 1.599 & 3.134 & 3.576 & 0.751 & 0.759 & 3.163 & 3.413 & 0.566 & 0.383 \\
\hline
\end{tabular}

\begin{tabular}{|c|c|c|c|c|c|c|c|c|c|c|c|c|c|}
\hline \multicolumn{2}{|c|}{$\overline{\mathrm{MA}}(1), \delta=0.5$} & \multicolumn{4}{|c|}{$\begin{array}{l}n=100 \\
n=10\end{array}$} & \multicolumn{4}{|c|}{$\begin{array}{l}n=400 \\
n\end{array}$} & \multicolumn{4}{|c|}{$\bar{n} n=1200$} \\
\hline$\overline{\bar{b}}$ & & $\overline{\bar{\sigma}}$ & $\overline{\overline{\text { mean }\left(\widehat{\sigma}^{*}\right)}}$ & $\overline{\overline{\mathrm{SD}\left(\widehat{\sigma}^{*}\right)}}$ & $\overline{\mathrm{MSE}\left(\widehat{\sigma}^{*}\right)}$ & $\overline{\bar{\sigma}}$ & $\overline{\text { mean }\left(\widehat{\sigma}^{*}\right)}$ & $\overline{\mathrm{SDD}\left(\widehat{\sigma}^{*}\right)}$ & $\overline{\mathrm{MSE}\left(\widehat{\sigma}^{*}\right)}$ & $\overline{\bar{\sigma}}$ & $\overline{\overline{\text { mean }\left(\widehat{\sigma}^{*}\right)}}$ & $\overline{\mathrm{S \textrm {SD }}\left(\widehat{\sigma}^{*}\right)}$ & $\overline{\mathrm{MSE}\left(\widehat{\sigma}^{*}\right)}$ \\
\hline \multirow[t]{4}{*}{$\bar{n}^{1 / 3}$} & $\widehat{A_{11}}$ & 3.443 & 3.801 & 0.527 & 0.406 & 3.347 & 3.653 & 0.368 & 0.229 & 3.386 & 3.526 & 0.297 & 0.108 \\
\hline & $\widehat{A}_{12}$ & 3.636 & 3.934 & 0.689 & 0.563 & 3.573 & 3.729 & 0.411 & 0.193 & 3.567 & 3.640 & 0.303 & 0.097 \\
\hline & $\widehat{A}_{21}$ & 4.110 & 4.346 & 0.731 & 0.590 & 4.026 & 4.263 & 0.449 & 0.258 & 4.007 & 4.158 & 0.341 & 0.139 \\
\hline & $\widehat{A}_{22}$ & 3.104 & 3.520 & 0.529 & 0.454 & 3.129 & 3.351 & 0.374 & 0.189 & 3.113 & 3.219 & 0.293 & 0.097 \\
\hline \multirow[t]{4}{*}{$3 n^{1 / 3}$} & $\widehat{A_{11}}$ & 3.443 & 4.043 & 0.981 & 1.322 & 3.347 & 3.782 & 0.661 & 0.627 & 3.386 & 3.625 & 0.456 & 0.265 \\
\hline & $\widehat{A}_{12}$ & 3.636 & 4.082 & 1.036 & 1.272 & 3.573 & 3.820 & 0.595 & 0.415 & 3.567 & 3.734 & 0.452 & 0.232 \\
\hline & $\widehat{A}_{21}$ & 4.110 & 4.666 & 1.213 & 1.780 & 4.026 & 4.389 & 0.745 & 0.688 & 4.007 & 4.252 & 0.492 & 0.302 \\
\hline & $\widehat{A}_{22}$ & 3.104 & 3.686 & 0.957 & 1.255 & 3.129 & 3.412 & 0.579 & 0.415 & 3.113 & 3.320 & 0.462 & 0.256 \\
\hline \multirow[t]{4}{*}{$5 n^{1 / 3}$} & $\widehat{A}_{11}$ & 3.443 & 4.035 & 1.190 & 1.766 & 3.347 & 3.918 & 0.854 & 1.057 & 3.386 & 3.710 & 0.613 & 0.481 \\
\hline & $\widehat{A}_{12}$ & 3.636 & 4.164 & 1.296 & 1.958 & 3.573 & 3.955 & 0.843 & 0.857 & 3.567 & 3.799 & 0.558 & 0.366 \\
\hline & $\widehat{A}_{21}$ & 4.11 & 4.522 & 1.421 & 2.189 & 4.026 & 4.511 & 0.941 & 1.121 & 4.007 & 4.314 & 0.640 & 0.504 \\
\hline & $\widehat{A}_{22}$ & 3.104 & 3.732 & 1.060 & 1.518 & 3.129 & 3.549 & 0.791 & 0.802 & 3.113 & 3.359 & 0.544 & 0.357 \\
\hline
\end{tabular}

\begin{tabular}{|c|c|c|c|c|c|c|c|c|c|c|c|c|c|}
\hline \multicolumn{2}{|c|}{$\mathrm{MA}(1), \delta=0.1$} & \multicolumn{4}{|c|}{$n=100$} & \multicolumn{4}{|c|}{$n=400$} & \multicolumn{4}{|c|}{$\begin{array}{l}n=1200 \\
\end{array}$} \\
\hline $\bar{b}$ & & $\overline{\bar{\sigma}}$ & $\overline{\text { mean }\left(\widehat{\sigma}^{*}\right)}$ & $\frac{1 \mathrm{SD}\left(\widehat{\sigma}^{*}\right)}{}$ & $\overline{\mathrm{MSE}\left(\widehat{\sigma}^{*}\right)}$ & $\overline{\bar{\sigma}}$ & $\overline{\text { mean }\left(\widehat{\sigma}^{*}\right)}$ & $\frac{1 \mathrm{SD}\left(\widehat{\sigma}^{*}\right)}{}$ & $\overline{\mathrm{MSE}\left(\widehat{\sigma}^{*}\right)}$ & $\overline{\bar{\sigma}}$ & $\overline{\text { mean }\left(\widehat{\sigma}^{*}\right)}$ & $\frac{1 \mathrm{SD}\left(\widehat{\sigma}^{*}\right)}{}$ & $\overline{\mathrm{MSE}\left(\widehat{\sigma}^{*}\right)}$ \\
\hline \multirow[t]{4}{*}{$\bar{n}^{1 / 3}$} & 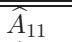 & 5.196 & (5.364 & 0.923 & 0.881 & 5.376 & 5.417 & 0.664 & 0.443 & 5.465 & "5.462 & 0.576 & 0.332 \\
\hline & $\widehat{A}_{12}$ & 3.396 & 3.727 & 0.630 & 0.507 & 3.413 & 3.588 & 0.400 & 0.191 & 3.399 & 3.524 & 0.304 & 0.108 \\
\hline & $\widehat{A}_{21}$ & 5.567 & 5.532 & 0.884 & 0.783 & 5.732 & 5.603 & 0.597 & 0.373 & 5.809 & 5.709 & 0.513 & 0.274 \\
\hline & $\widehat{A}_{22}$ & 3.112 & 3.400 & 0.512 & 0.345 & 3.127 & 3.294 & 0.379 & 0.172 & 3.123 & 3.232 & 0.298 & 0.101 \\
\hline \multirow[t]{4}{*}{$3 n^{1 / 3}$} & $\widehat{A_{11}}$ & 5.196 & 5.934 & 1.585 & 3.058 & 5.376 & 5.793 & 1.143 & 1.481 & 5.465 & 5.715 & 0.969 & 1.002 \\
\hline & $\widehat{A}_{12}$ & 3.396 & 3.959 & 0.986 & 1.289 & 3.413 & 3.799 & 0.628 & 0.543 & 3.399 & 3.631 & 0.457 & 0.263 \\
\hline & $\widehat{A}_{21}$ & 5.567 & 6.053 & 1.466 & 2.386 & 5.732 & 5.949 & 0.967 & 0.983 & 5.809 & 5.943 & 0.818 & 0.687 \\
\hline & $\widehat{A}_{22}$ & 3.112 & 3.611 & 0.918 & 1.092 & 3.127 & 3.498 & 0.678 & 0.598 & 3.123 & 3.337 & 0.474 & 0.271 \\
\hline \multirow[t]{4}{*}{$5 n^{1 / 3}$} & $\widehat{A_{11}}$ & 5.196 & 6.033 & 1.859 & 4.156 & 5.376 & 6.147 & 1.497 & 2.835 & 5.465 & 5.946 & 1.219 & 1.718 \\
\hline & $\widehat{A}_{12}$ & 3.396 & 3.950 & 1.198 & 1.742 & 3.413 & 3.876 & 0.823 & 0.892 & 3.399 & 3.671 & 0.582 & 0.413 \\
\hline & $\widehat{A}_{21}$ & 5.567 & 6.046 & 1.767 & 3.353 & 5.732 & 6.150 & 1.163 & 1.528 & 5.809 & 6.086 & 0.959 & 0.997 \\
\hline & $\widehat{A}_{22}$ & 3.112 & 3.583 & 1.083 & 1.396 & 3.127 & 3.486 & 0.788 & 0.749 & 3.123 & 3.342 & 0.566 & 0.369 \\
\hline
\end{tabular}

TABLE 1. Standard deviations $\widehat{\sigma}^{*}$ of the entries of $n\left(\widehat{A}-I_{2}\right)$ estimated by the RBB bootstrap for the $\operatorname{VMA}(1)$ model a), sample sizes $n \in\{100,400,1200\}$, (rounded) block lengths $b \in\left\{n^{1 / 3}, 3 n^{1 / 3}, 5 n^{1 / 3}\right\}$ and $\delta \in\{0.9,0.5,0.1\}$. The true parameter $\sigma$ is estimated by 20,000 Monte Carlo replications.

b) $\operatorname{VAR}(1)$ model $U_{2 t}=\Psi U_{2, t-1}+\epsilon_{t}$

where $\epsilon_{t} \sim \mathcal{N}(0, \Sigma)$. The parameter specifications $B=(0.5,-0.5)$,

$$
\Psi=\left(\begin{array}{cc}
\delta & -0.4 \\
0 & 0.5
\end{array}\right) \quad \text { and } \quad \Sigma=\left(\begin{array}{cc}
1 & 0.5 \\
0.5 & 1
\end{array}\right)
$$




\begin{tabular}{|c|c|c|c|c|c|c|c|c|c|c|c|c|c|}
\hline \multicolumn{2}{|c|}{ 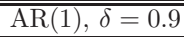 } & \multicolumn{4}{|c|}{$n=100$} & \multicolumn{4}{|c|}{$n=400$} & \multicolumn{4}{|c|}{$n=1200$} \\
\hline 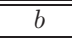 & & 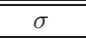 & $\overline{\mathrm{m} e a n}\left(\widehat{\sigma}^{*}\right)$ & $\overline{\mathrm{SD}\left(\hat{\sigma}^{*}\right)}$ & $\operatorname{MSE}\left(\widehat{\sigma}^{*}\right)$ & 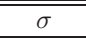 & $\overline{\operatorname{mean}\left(\widehat{\sigma}^{*}\right)}$ & $\overline{\mathrm{SD}\left(\widehat{\sigma}^{*}\right)}$ & $\operatorname{MSE}\left(\widehat{\sigma}^{*}\right)$ & 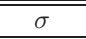 & "mean $\left(\hat{\sigma}^{*}\right)$ & $\mathrm{SD}\left(\hat{\sigma}^{*}\right)$ & $\operatorname{MSE}\left(\widehat{\sigma}^{*}\right)$ \\
\hline \multirow[t]{4}{*}{$n^{1 / 3}$} & $\widehat{A_{11}}$ & 2.431 & 2.362 & 0.267 & 0.076 & 2.491 & 2.064 & 0.140 & 0.202 & 2.487 & 2.037 & 0.136 & 0.221 \\
\hline & $\widehat{A}_{12}$ & 11.981 & 7.771 & 2.033 & 21.864 & 15.237 & 8.749 & 1.358 & 43.936 & 16.539 & 10.324 & 1.112 & 39.866 \\
\hline & $\widehat{A}_{21}$ & 1.081 & 1.736 & 0.478 & 0.656 & 0.861 & 1.308 & 0.210 & 0.244 & 0.807 & 1.081 & 0.120 & 0.089 \\
\hline & $\widehat{A}_{22}$ & 2.672 & 2.919 & 0.417 & 0.235 & 2.566 & 2.521 & 0.214 & 0.048 & 2.504 & 2.413 & 0.154 & 0.032 \\
\hline \multirow[t]{4}{*}{$3 n^{1 / 3}$} & $\widehat{A_{11}}$ & 2.431 & 2.497 & 0.512 & 0.266 & 2.491 & 2.341 & 0.354 & 0.148 & 2.487 & 2.352 & 0.315 & 0.117 \\
\hline & $\widehat{A}_{12}$ & 11.981 & 10.064 & 3.406 & 15.279 & 15.237 & 12.645 & 2.721 & 14.126 & 16.539 & 14.134 & 2.159 & 10.445 \\
\hline & $\widehat{A}_{21}$ & 1.081 & 1.487 & 0.530 & 0.445 & 0.861 & 1.035 & 0.236 & 0.086 & 0.807 & 0.911 & 0.135 & 0.029 \\
\hline & $\widehat{A}_{22}$ & 2.672 & 3.030 & 0.653 & 0.555 & 2.566 & 2.648 & 0.337 & 0.120 & 2.504 & 2.541 & 0.224 & 0.051 \\
\hline \multirow[t]{4}{*}{$5 n^{1 / 3}$} & $\widehat{A_{11}}$ & 2.431 & 2.611 & 0.687 & 0.504 & 2.491 & 2.563 & 0.530 & 0.286 & 2.487 & 2.541 & 0.441 & 0.197 \\
\hline & $\widehat{A}_{12}$ & 11.981 & 11.149 & 4.535 & 21.264 & 15.237 & 13.960 & 3.356 & 12.894 & 16.539 & 15.627 & 2.79 & 8.614 \\
\hline & $\widehat{A}_{21}$ & 1.081 & 1.424 & 0.641 & 0.529 & 0.861 & 1.030 & 0.257 & 0.095 & 0.807 & 0.897 & 0.164 & 0.035 \\
\hline & $\widehat{A}_{22}$ & 2.672 & 3.196 & 0.892 & 1.070 & 2.566 & 2.796 & 0.436 & 0.243 & 2.504 & 2.666 & 0.303 & 0.118 \\
\hline
\end{tabular}

\begin{tabular}{|c|c|c|c|c|c|c|c|c|c|c|c|c|c|}
\hline \multicolumn{2}{|c|}{$\overline{\mathrm{ARR}(1), \delta=0.5}$} & \multicolumn{4}{|c|}{$=100$} & \multicolumn{4}{|c|}{$n=400$} & \multicolumn{4}{|c|}{$=n=1200$} \\
\hline$\overline{c b}$ & & $\overline{\sigma \sigma}$ & "mean $\left(\widehat{\sigma}^{*}\right)$ & 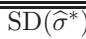 & $\overline{\mathrm{MSSE}\left(\widehat{\sigma}^{*}\right)}$ & 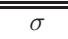 & $\overline{\mathrm{emean}\left(\widehat{\sigma}^{*}\right)}$ & $\overline{\mathrm{SDD}\left(\widehat{\sigma}^{*}\right)}$ & 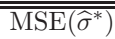 & $\overline{\sigma \sigma}$ & $\overline{\mathrm{c} \text { mean }\left(\widehat{\sigma}^{*}\right)}$ & $\overline{\mathrm{SDD}\left(\widehat{\sigma}^{*}\right)}$ & 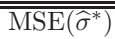 \\
\hline \multirow[t]{4}{*}{$n^{1 / 3}$} & $\overline{\widehat{A}_{11}}$ & 3.669 & 3.419 & 0.465 & 0.279 & "4.060 & 3.472 & 0.352 & 0.469 & 4.219 & 3.716 & 0.365 & 0.386 \\
\hline & $\widehat{A}_{12}$ & 4.196 & 4.108 & 0.812 & 0.667 & 4.557 & 4.138 & 0.552 & 0.480 & 4.706 & 4.323 & 0.472 & 0.370 \\
\hline & $\widehat{A}_{21}$ & 3.858 & 3.761 & 0.720 & 0.528 & 4.094 & 3.717 & 0.429 & 0.326 & 4.16 & 3.853 & 0.358 & 0.223 \\
\hline & $\widehat{A}_{22}$ & 2.608 & 2.840 & 0.359 & 0.183 & 2.658 & 2.632 & 0.213 & 0.046 & 2.686 & 2.582 & 0.176 & 0.042 \\
\hline \multirow[t]{4}{*}{$3 n^{1 / 3}$} & $\widehat{A_{11}}$ & 3.669 & 4.011 & 0.978 & 1.072 & 4.060 & 4.123 & 0.822 & 0.679 & 4.219 & 4.207 & 0.689 & 0.475 \\
\hline & $\widehat{A}_{12}$ & 4.196 & 4.665 & 1.296 & 1.900 & 4.557 & 4.704 & 1.043 & 1.109 & 4.706 & 4.709 & 0.810 & 0.656 \\
\hline & $\widehat{A}_{21}$ & 3.858 & 4.095 & 1.065 & 1.191 & 4.094 & 4.101 & 0.799 & 0.639 & 4.16 & 4.194 & 0.652 & 0.427 \\
\hline & $\widehat{A}_{22}$ & 2.608 & 3.033 & 0.668 & 0.627 & 2.658 & 2.805 & 0.378 & 0.164 & 2.686 & 2.747 & 0.285 & 0.085 \\
\hline \multirow[t]{4}{*}{$5 n^{1 / 3}$} & $\widehat{A_{11}}$ & 3.669 & 4.164 & 1.146 & 1.558 & 4.060 & 4.351 & 1.118 & 1.336 & 4.219 & 4.425 & 0.875 & 0.807 \\
\hline & $\widehat{A}_{12}$ & 4.196 & 4.626 & 1.624 & 2.823 & 4.557 & 4.967 & 1.373 & 2.054 & 4.706 & 4.939 & 1.014 & 1.083 \\
\hline & $\widehat{A}_{21}$ & 3.858 & 4.147 & 1.299 & 1.770 & 4.094 & 4.294 & 1.036 & 1.113 & 4.160 & 4.293 & 0.760 & 0.595 \\
\hline & $\widehat{A}_{22}$ & 2.608 & 3.013 & 0.749 & 0.725 & 2.658 & 2.962 & 0.519 & 0.362 & 2.686 & 2.859 & 0.380 & 0.174 \\
\hline
\end{tabular}

\begin{tabular}{|c|c|c|c|c|c|c|c|c|c|c|c|c|c|}
\hline \multicolumn{2}{|c|}{$\overline{\mathrm{AR}(1), \delta=0.1}$} & \multicolumn{4}{|c|}{$\bar{c} n=100$} & \multicolumn{4}{|c|}{$\bar{c} n=400$} & \multicolumn{4}{|c|}{ 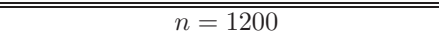 } \\
\hline 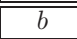 & & $\overline{\sigma \sigma}$ & mean $\left(\widehat{\sigma}^{*}\right)$ & 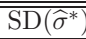 & 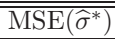 & 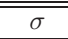 & "mean $\left(\widehat{\sigma}^{*}\right)$ & 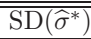 & 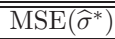 & $\overline{\bar{\sigma} \sigma}$ & 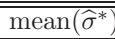 & $\overline{\mathrm{SDD}\left(\widehat{\sigma}^{*}\right)}$ & $\overline{\mathrm{M}} \operatorname{\mathrm {MSE}}\left(\widehat{\sigma}^{*}\right)$ \\
\hline \multirow[t]{4}{*}{$\bar{c} n^{1 / 3}$} & $\widehat{\overline{A_{11}}}$ & "6.134 & 5.347 & 0.864 & 1.366 & 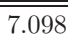 & 5.943 & 0.741 & 1.882 & 7.324 & 6.478 & 0.672 & 1.169 \\
\hline & $\widehat{A}_{12}$ & 3.497 & 3.703 & 0.675 & 0.498 & 3.748 & 3.594 & 0.472 & 0.247 & 3.813 & 3.662 & 0.389 & 0.174 \\
\hline & $\widehat{A}_{21}$ & 6.556 & 5.420 & 0.892 & 2.084 & 7.565 & 6.100 & 0.687 & 2.617 & 7.601 & 6.691 & 0.628 & 1.222 \\
\hline & $\widehat{A}_{22}$ & 2.615 & 2.820 & 0.318 & 0.143 & 2.769 & 2.678 & 0.208 & 0.052 & 2.786 & 2.691 & 0.191 & 0.045 \\
\hline \multirow[t]{4}{*}{$3 n^{1 / 3}$} & $\widehat{A_{11}}$ & 6.134 & 6.563 & 1.536 & 2.543 & 7.098 & 7.241 & 1.438 & 2.088 & 7.324 & 7.388 & 1.228 & 1.511 \\
\hline & $\widehat{A}_{12}$ & 3.497 & 3.985 & 1.153 & 1.567 & 3.748 & 3.982 & 0.827 & 0.740 & 3.813 & 3.937 & 0.682 & 0.480 \\
\hline & $\widehat{A}_{21}$ & 6.556 & 6.576 & 1.698 & 2.883 & 7.565 & 7.369 & 1.381 & 1.946 & 7.601 & 7.588 & 1.151 & 1.325 \\
\hline & $\widehat{A}_{22}$ & 2.615 & 2.981 & 0.614 & 0.511 & 2.769 & 2.937 & 0.422 & 0.206 & 2.786 & 2.860 & 0.317 & 0.106 \\
\hline \multirow[t]{4}{*}{$5 n^{1 / 3}$} & $\widehat{A}_{11}$ & 6.134 & 6.805 & 1.784 & 3.634 & 7.098 & 7.579 & 1.869 & 3.724 & 7.324 & 7.616 & 1.519 & 2.391 \\
\hline & $\widehat{A}_{12}$ & 3.497 & 4.097 & 1.374 & 2.248 & 3.748 & 4.035 & 0.949 & 0.983 & 3.813 & 4.006 & 0.782 & 0.650 \\
\hline & $\widehat{A}_{21}$ & 6.556 & 6.744 & 2.067 & 4.306 & 7.565 & 7.836 & 1.848 & 3.489 & 7.601 & 7.806 & 1.466 & 2.190 \\
\hline & $\widehat{A}_{22}$ & 2.615 & 3.071 & 0.809 & 0.862 & 2.769 & 3.030 & 0.520 & 0.338 & 2.786 & 2.949 & 0.402 & 0.188 \\
\hline
\end{tabular}

TABLE 2. Standard deviations $\widehat{\sigma}^{*}$ of the entries of $n\left(\widehat{A}-I_{2}\right)$ estimated by the RBB bootstrap for the $\operatorname{VAR}(1)$ model b), sample sizes $n \in\{100,400,1200\}$, (rounded) block lengths $b \in\left\{n^{1 / 3}, 3 n^{1 / 3}, 5 n^{1 / 3}\right\}$ and $\delta \in\{0.9,0.5,0.1\}$. The true parameter $\sigma$ is estimated by 20,000 Monte Carlo replications.

are used in both cases a) and b) with $\delta \in\{0.1,0.5,0.9\} . \quad\left\{e_{t}\right\}$ and $\left\{\epsilon_{t}\right\}$ are independent. By using the notation of Section 2.1, this setup corresponds to the cointegration model (3.1) with $m_{1}=1$ and $m_{2}=2$.

Further, note that the bivariate process $\left\{X_{2 t}\right\}$ is (purely) integrated and of the form (2.1) and that $\left\{U_{1 t}\right\}$ and $\left\{U_{2 t}\right\}$ are independent by construction. Hence, an application of the RBB scheme 


\begin{tabular}{|c|c|c|c|c|c|c|c|c|c|c|c|c|c|}
\hline \multicolumn{2}{|c|}{$\mathrm{MA}(1), \delta=0.9$} & \multicolumn{4}{|c|}{$n=100$} & \multicolumn{4}{|c|}{$n=400$} & \multicolumn{4}{|c|}{$\bar{n} n=1200$} \\
\hline 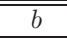 & & 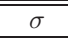 & $\overline{\operatorname{mean}\left(\widehat{\sigma}^{*}\right)}$ & $\overline{\mathrm{SD}\left(\widehat{\sigma}^{*}\right)}$ & $\overline{\mathrm{MSE}\left(\widehat{\sigma}^{*}\right)}$ & $\bar{\sigma} \sigma$ & mean $\left(\widehat{\sigma}^{*}\right)$ & $\overline{\mathrm{SSD}\left(\hat{\sigma}^{*}\right)}$ & $\operatorname{MSE}\left(\widehat{\sigma}^{*}\right)$ & $\bar{\sigma}$ & "mean $\left(\widehat{\sigma}^{*}\right)$ & $\overline{\mathrm{SD}\left(\hat{\sigma}^{*}\right)}$ & $\overline{\mathrm{MSE}\left(\hat{\sigma}^{*}\right)}$ \\
\hline \multirow[t]{2}{*}{$n^{1 / 3}$} & $\widehat{\alpha}$ & 0.805 & 0.714 & 0.109 & 0.020 & 0.792 & 0.755 & 0.068 & 0.006 & 0.798 & 0.763 & 0.054 & 0.004 \\
\hline & $\widehat{\beta}$ & 0.714 & 0.687 & 0.135 & 0.019 & 0.695 & 0.694 & 0.088 & 0.008 & 0.698 & 0.688 & 0.061 & 0.004 \\
\hline \multirow[t]{2}{*}{$3 n^{1 / 3}$} & $\widehat{\alpha}$ & 0.805 & 0.662 & 0.150 & 0.043 & 0.792 & 0.750 & 0.113 & 0.015 & 0.798 & 0.771 & 0.080 & 0.007 \\
\hline & $\widehat{\beta}$ & 0.714 & 0.643 & 0.188 & 0.040 & 0.695 & 0.692 & 0.135 & 0.018 & 0.698 & 0.690 & 0.093 & 0.009 \\
\hline \multirow[t]{2}{*}{$5 n^{1 / 3}$} & $\widehat{\alpha}$ & 0.805 & 0.610 & 0.189 & 0.074 & 0.792 & 0.716 & 0.140 & 0.025 & 0.798 & 0.749 & 0.099 & 0.012 \\
\hline & $\widehat{\beta}$ & 0.714 & 0.604 & 0.222 & 0.061 & 0.695 & 0.668 & 0.172 & 0.030 & 0.698 & 0.680 & 0.117 & 0.014 \\
\hline
\end{tabular}

\begin{tabular}{|c|c|c|c|c|c|c|c|c|c|c|c|c|c|}
\hline \multicolumn{2}{|c|}{$\overline{\mathrm{MA}}(1), \delta=0.5$} & \multicolumn{4}{|c|}{$\bar{c} n=100$} & \multicolumn{4}{|c|}{$\bar{c} n=400$} & \multicolumn{4}{|c|}{$\bar{n} n=1200$} \\
\hline$\overline{\bar{b}}$ & & $\overline{\bar{\sigma} \sigma}$ & $\overline{\mathrm{p} \text { mean }\left(\widehat{\sigma}^{*}\right)}$ & $\overline{\overline{\mathrm{SD}\left(\widehat{\sigma}^{*}\right)}}$ & $\overline{\mathrm{MSE}\left(\widehat{\sigma}^{*}\right)}$ & $\overline{\sigma \sigma}$ & $\overline{\text { mean }\left(\widehat{\sigma}^{*}\right)}$ & $\overline{\overline{\mathrm{SD}\left(\widehat{\sigma}^{*}\right)}}$ & 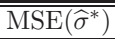 & $\overline{\sigma \sigma}$ & $\overline{\text { mean }\left(\widehat{\sigma}^{*}\right)}$ & $\overline{\overline{\mathrm{SD}\left(\widehat{\sigma}^{*}\right)}}$ & 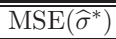 \\
\hline \multirow[t]{2}{*}{$\bar{c} n^{1 / 3}$} & $\widehat{\alpha}$ & 0.631 & 0.580 & 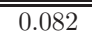 & 0.009 & 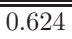 & 0.598 & (0.053 & 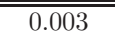 & 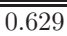 & 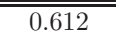 & 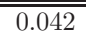 & 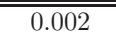 \\
\hline & $\widehat{\beta}$ & 0.572 & 0.559 & 0.108 & 0.012 & 0.552 & 0.548 & 0.063 & 0.004 & 0.547 & 0.549 & 0.049 & 0.002 \\
\hline \multirow[t]{2}{*}{$3 n^{1 / 3}$} & $\widehat{\alpha}$ & 0.631 & 0.535 & 0.128 & 0.025 & 0.624 & 0.584 & 0.085 & 0.009 & 0.629 & 0.607 & 0.063 & 0.004 \\
\hline & $\widehat{\beta}$ & 0.572 & 0.510 & 0.151 & 0.027 & 0.552 & 0.536 & 0.102 & 0.011 & 0.547 & 0.543 & 0.075 & 0.006 \\
\hline \multirow[t]{2}{*}{$5 n^{1 / 3}$} & $\widehat{\alpha}$ & 0.631 & 0.493 & 0.160 & 0.045 & 0.624 & 0.563 & 0.115 & 0.017 & 0.629 & 0.598 & 0.079 & 0.007 \\
\hline & $\widehat{\beta}$ & 0.572 & 0.487 & 0.192 & 0.044 & 0.552 & 0.527 & 0.141 & 0.020 & 0.547 & 0.542 & 0.094 & 0.009 \\
\hline
\end{tabular}

\begin{tabular}{|c|c|c|c|c|c|c|c|c|c|c|c|c|c|}
\hline \multicolumn{2}{|c|}{$\overline{\mathrm{MA}(1), \delta=0.1}$} & \multicolumn{4}{|c|}{$\bar{n} n=100$} & \multicolumn{4}{|c|}{$n=400$} & \multicolumn{4}{|c|}{$n=1200$} \\
\hline 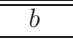 & & 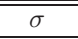 & $\overline{\operatorname{mean}\left(\widehat{\sigma}^{*}\right)}$ & $\overline{\mathrm{SD}\left(\widehat{\sigma}^{*}\right)}$ & 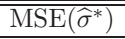 & $\overline{\sigma \sigma}$ & mean $\left(\widehat{\sigma}^{*}\right)$ & $\overline{\mathrm{SD}\left(\widehat{\sigma}^{*}\right)}$ & $\operatorname{MSE}\left(\widehat{\sigma}^{*}\right)$ & $\overline{\sigma \sigma}$ & mean $\left(\widehat{\sigma}^{*}\right)$ & $\overline{\mathrm{SD}\left(\widehat{\sigma}^{*}\right)}$ & $\operatorname{MSSE}\left(\hat{\sigma}^{*}\right)$ \\
\hline \multirow[t]{2}{*}{$n^{1 / 3}$} & $\overline{\widehat{\alpha} \widehat{\alpha}}$ & 0.466 & 0.450 & 0.059 & 0.004 & 0.463 & 0.459 & 0.038 & 0.001 & 0.460 & 0.459 & 0.033 & 0.001 \\
\hline & $\widehat{\beta}$ & 0.412 & 0.430 & 0.079 & 0.007 & 0.403 & 0.422 & 0.048 & 0.003 & 0.399 & 0.412 & 0.036 & 0.001 \\
\hline \multirow[t]{2}{*}{$3 n^{1 / 3}$} & $\widehat{\alpha}$ & 0.466 & 0.402 & 0.099 & 0.014 & 0.463 & 0.438 & 0.061 & 0.004 & 0.460 & 0.449 & 0.047 & 0.002 \\
\hline & $\widehat{\beta}$ & 0.412 & 0.389 & 0.121 & 0.015 & 0.403 & 0.408 & 0.075 & 0.006 & 0.399 & 0.406 & 0.056 & 0.003 \\
\hline \multirow[t]{2}{*}{$5 n^{1 / 3}$} & $\widehat{\alpha}$ & 0.466 & 0.365 & 0.111 & 0.022 & 0.463 & 0.419 & 0.078 & 0.008 & 0.460 & 0.439 & 0.060 & 0.004 \\
\hline & $\widehat{\beta}$ & 0.412 & 0.350 & 0.134 & 0.022 & 0.403 & 0.390 & 0.092 & 0.009 & 0.399 & 0.396 & 0.070 & 0.005 \\
\hline
\end{tabular}

TABLE 3. Standard deviations $\widehat{\sigma}^{*}$ of $n^{-1 / 2} \widehat{\alpha}$ and $\widehat{\beta}$ estimated by the CBB bootstrap for the $\operatorname{VMA}(1)$ model a), sample sizes $n \in\{100,400,1200\}$, (rounded) block lengths $b \in\left\{n^{1 / 3}, 3 n^{1 / 3}, 5 n^{1 / 3}\right\}$ and $\delta \in\{0.9,0.5,0.1\}$. The true parameter $\sigma$ is estimated by 20,000 Monte Carlo replications.

as proposed in Section 3.2 leads to trivariate bootstrap data $X_{t}^{+}=\left(X_{1 t}^{+}, X_{2 t}^{+\prime}\right)^{\prime}, t=1, \ldots, n$ such that the bivariate observations $X_{2 t}^{+}$can be regarded as being generated by the CBB defined in Section 2.2. That is, we have $X_{2 t}^{+}=X_{2 t}^{*}, t=1, \ldots, n$. This observation allows us to investigate jointly the finite sample performance of the RBB and CBB by using the setup described above.

For the trivariate cointegrated model in (4.1) and (4.2), we consider the estimator $\widehat{B}$ defined in (3.2) and for the purely integrated bivariate sub-model (4.2), we consider the estimator $\widehat{A}$ defined in (2.7) which is obtained from regressing $X_{2 t}$ on $X_{2, t-1}$ as well as the estimators $\widehat{\alpha}$ and $\widehat{\beta}$ defined in (2.10) obtained from regressing the first coordinate of $X_{2 t}$ on its second coordinate.

To judge the finite sample performance of $\mathrm{RBB}$ and $\mathrm{CBB}$, the task is to estimate the standard deviations of (the entries of $n(\widehat{B}-B), n\left(\widehat{A}-I_{2}\right), n^{-1 / 2} \widehat{\alpha}$ and $\widehat{\beta}$. Further, we compute coverage rates of $95 \%$ bootstrap confidence intervals for (the entries) of $B$. We show the results for $n \in\{100,400,1200\}$ and to illustrate how sensitive the bootstrap reacts on the choice of the block length $b$, we show results for three (rounded) values of $b \in\left\{n^{1 / 3}, 3 n^{1 / 3}, 5 n^{1 / 3}\right\}$. For both settings a) and b) above, we generate $T=500$ time series and we use $K=500$ bootstrap replicates to estimate the standard deviations and to construct the confidence intervals. The true parameter $\sigma$ is estimated by 20,000 Monte Carlo replications.

In Figure 1, we show realizations of the model (4.1)-(4.2) for both cases a) and b), $\delta \in$ 


\begin{tabular}{|c|c|c|c|c|c|c|c|c|c|c|c|c|c|}
\hline \multicolumn{2}{|c|}{$\overline{\mathrm{AR}}(1), \delta=0.9$} & \multicolumn{4}{|c|}{$n=100$} & \multicolumn{4}{|c|}{$\overline{n=400}$} & \multicolumn{4}{|c|}{$n=1200$} \\
\hline$b$ & & $\sigma$ & mean $\left(\widehat{\sigma}^{*}\right)$ & $\overline{\mathrm{SD}\left(\widehat{\sigma}^{*}\right)}$ & $\overline{\mathrm{MSSE}\left(\hat{\sigma}^{*}\right)}$ & $\overline{\sigma \sigma}$ & (mean $\left(\widehat{\sigma}^{*}\right)$ & 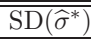 & 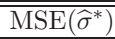 & $\sigma$ & 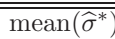 & $\overline{\mathrm{SD}\left(\widehat{\sigma}^{*}\right)}$ & $\overline{\mathrm{MSSE}\left(\widehat{\sigma}^{*}\right)}$ \\
\hline \multirow[t]{2}{*}{ 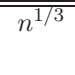 } & $\widehat{\alpha}$ & 3.499 & 1.766 & 0.442 & 3.201 & 3.919 & 2.282 & 0.321 & 2.781 & 4.099 & 2.780 & 0.253 & 1.804 \\
\hline & $\widehat{\beta}$ & 2.804 & 1.453 & 0.414 & 1.995 & 2.792 & 1.693 & 0.272 & 1.282 & 2.754 & 1.979 & 0.212 & 0.645 \\
\hline \multirow[t]{2}{*}{$3 n^{1 / 3}$} & $\widehat{\alpha}$ & 3.499 & 2.234 & 0.717 & 2.116 & 3.919 & 3.152 & 0.627 & 0.982 & 4.099 & 3.525 & 0.436 & 0.519 \\
\hline & $\widehat{\beta}$ & 2.804 & 1.765 & 0.666 & 1.522 & 2.792 & 2.257 & 0.525 & 0.561 & 2.754 & 2.425 & 0.358 & 0.237 \\
\hline \multirow[t]{2}{*}{$5 n^{1 / 3}$} & $\widehat{\alpha}$ & 3.499 & 2.287 & 0.948 & 2.369 & 3.919 & 3.238 & 0.698 & 0.951 & 4.099 & 3.643 & 0.515 & 0.473 \\
\hline & $\widehat{\beta}$ & 2.804 & 1.819 & 0.886 & 1.756 & 2.792 & 2.286 & 0.574 & 0.586 & 2.754 & 2.524 & 0.452 & 0.257 \\
\hline
\end{tabular}

\begin{tabular}{|c|c|c|c|c|c|c|c|c|c|c|c|c|c|}
\hline \multicolumn{2}{|c|}{$\overline{\mathrm{AR}}(1), \delta=0.5$} & \multicolumn{4}{|c|}{$n=100$} & \multicolumn{4}{|c|}{$n=400$} & \multicolumn{4}{|c|}{$\bar{c} n=1200$} \\
\hline 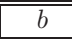 & & 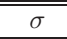 & mean $\left(\widehat{\sigma}^{*}\right)$ & $\overline{\mathrm{SD}\left(\widehat{\sigma}^{*}\right)}$ & $\overline{\mathrm{MSSE}\left(\widehat{\sigma}^{*}\right)}$ & 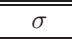 & mean $\left(\widehat{\sigma}^{*}\right)$ & $\overline{\mathrm{SD}\left(\widehat{\sigma}^{*}\right)}$ & 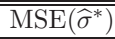 & $\overline{\bar{\sigma} \sigma}$ & $\overline{\mathrm{c} \operatorname{mean}\left(\widehat{\sigma}^{*}\right)}$ & $\overline{\mathrm{SD}\left(\widehat{\sigma}^{*}\right)}$ & $\overline{\mathrm{MSSE}\left(\widehat{\sigma}^{*}\right)}$ \\
\hline \multirow[t]{2}{*}{$n^{1 / 3}$} & $\overline{\bar{\alpha}}$ & 0.827 & 0.764 & "0.119 & 0.018 & 0.829 & 0.799 & 0.074 & 0.006 & 0.834 & 0.817 & 0.054 & 0.003 \\
\hline & $\widehat{\beta}$ & 0.593 & 0.627 & 0.126 & 0.017 & 0.558 & 0.603 & 0.075 & 0.008 & 0.552 & 0.578 & 0.052 & 0.003 \\
\hline \multirow[t]{2}{*}{$3 n^{1 / 3}$} & $\widehat{\alpha}$ & 0.827 & 0.710 & 0.181 & 0.046 & 0.829 & 0.786 & 0.117 & 0.016 & 0.834 & 0.810 & 0.089 & 0.009 \\
\hline & $\widehat{\beta}$ & 0.593 & 0.566 & 0.180 & 0.033 & 0.558 & 0.566 & 0.116 & 0.014 & 0.552 & 0.553 & 0.083 & 0.007 \\
\hline \multirow[t]{2}{*}{$5 n^{1 / 3}$} & $\widehat{\alpha}$ & 0.827 & 0.638 & 0.204 & 0.077 & 0.829 & 0.761 & 0.150 & 0.027 & 0.834 & 0.795 & 0.104 & 0.012 \\
\hline & $\widehat{\beta}$ & 0.593 & 0.497 & 0.193 & 0.047 & 0.558 & 0.546 & 0.141 & 0.020 & 0.552 & 0.546 & 0.092 & 0.008 \\
\hline
\end{tabular}

\begin{tabular}{|c|c|c|c|c|c|c|c|c|c|c|c|c|c|}
\hline \multicolumn{2}{|c|}{$\mathrm{AR}(1), \delta=0.1$} & \multicolumn{4}{|c|}{$n=100$} & \multicolumn{4}{|c|}{$n=400$} & \multicolumn{4}{|c|}{$n=1200$} \\
\hline 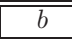 & & 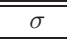 & "mean $\left(\widehat{\sigma}^{*}\right)$ & $\overline{\mathrm{SDD}\left(\widehat{\sigma}^{*}\right)}$ & 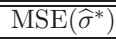 & 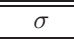 & 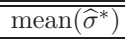 & $\overline{\mathrm{SDD}\left(\widehat{\sigma}^{*}\right)}$ & 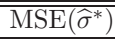 & 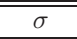 & 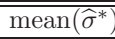 & $\overline{\mathrm{SDD}\left(\widehat{\sigma}^{*}\right)}$ & $\overline{\mathrm{MSE}\left(\widehat{\sigma}^{*}\right)}$ \\
\hline \multirow[t]{2}{*}{$n^{1 / 3}$} & $\widehat{\overline{\alpha \alpha}}$ & 0.471 & 0.498 & "0.068 & 0.005 & 0.464 & 0.492 & 0.040 & 0.002 & 0.461 & 0.484 & 0.031 & 0.001 \\
\hline & $\widehat{\beta}$ & 0.332 & 0.414 & 0.076 & 0.013 & 0.308 & 0.369 & 0.044 & 0.006 & 0.303 & 0.342 & 0.032 & 0.003 \\
\hline \multirow[t]{2}{*}{$3 n^{1 / 3}$} & $\widehat{\alpha}$ & 0.471 & 0.417 & 0.095 & 0.012 & 0.464 & 0.449 & 0.063 & 0.004 & 0.461 & 0.458 & 0.045 & 0.002 \\
\hline & $\widehat{\beta}$ & 0.332 & 0.334 & 0.106 & 0.011 & 0.308 & 0.322 & 0.060 & 0.004 & 0.303 & 0.315 & 0.044 & 0.002 \\
\hline \multirow[t]{2}{*}{$5 n^{1 / 3}$} & $\widehat{\alpha}$ & 0.471 & 0.371 & 0.106 & 0.021 & 0.464 & 0.427 & 0.079 & 0.008 & 0.461 & 0.451 & 0.056 & 0.003 \\
\hline & $\widehat{\beta}$ & 0.332 & 0.297 & 0.114 & 0.014 & 0.308 & 0.305 & 0.073 & 0.005 & 0.303 & 0.308 & 0.053 & 0.003 \\
\hline
\end{tabular}

TABLE 4. Standard deviations $\widehat{\sigma}^{*}$ of $n^{-1 / 2} \widehat{\alpha}$ and $\widehat{\beta}$ estimated by the CBB bootstrap for the $\operatorname{VAR}(1)$ model b), sample sizes $n \in\{100,400,1200\}$, (rounded) block lengths $b \in\left\{n^{1 / 3}, 3 n^{1 / 3}, 5 n^{1 / 3}\right\}$ and $\delta \in\{0.9,0.5,0.1\}$. The true parameter $\sigma$ is estimated by 20,000 Monte Carlo replications.

$\{0.9,0.5,0.1\}$ and sample size $n=400$. These figures clearly indicate the cointegration relation. In Tables 1-6, we present the simulation results for the task of (CBB and RBB) bootstrap estimation of standard deviations. In each table, we show the true standard deviations $\sigma$ together with averages of their bootstrap estimates mean $\left(\widehat{\sigma}^{*}\right)$, their standard deviations $S D\left(\widehat{\sigma}^{*}\right)$ and the corresponding mean squared errors $M S E\left(\widehat{\sigma}^{*}\right)$.

The overall performance appears to be quite well, where the results do not seem to react very sensitive on the choice of the block length. In general a larger block length tends to be superior in the situation, where more dependence structure has to be captured by the block bootstrap which is the case for the AR-type model. In particular, in almost all cases, the results show an improving behavior wrt MSE for increasing sample size. Essentially, the only exception is the case of $\widehat{A}_{12}$ in the upper panel of Table 2. In this case, although the true parameter $\sigma$ is converging, it is still increasing considerably from 11.981 to 16.539 from $n=400$ to $n=1200$. Here, in comparison to $b \in\left\{3 n^{1 / 3}, 5 n^{1 / 3}\right\}$, the block length $b=n^{1 / 3}$ is just too small which results in a large bias while the standard deviation behaves quite well. This means, the large MSE is mainly caused by a large bias, which appears to be a problem of the sample size. For larger block lengths $b \in\left\{3 n^{1 / 3}, 5 n^{1 / 3}\right\}$, the approximation of $\sigma$ is superior which leads to a smaller bias and, consequently, to a smaller MSE. Moreover, the simulation results show that different block lengths $b$ turn out to be optimal for the different parameters. For example, in Table 2, upper panel, for $n=1200$, it can be seen that $b=n^{1 / 3}$ performs best for $\widehat{A}_{22}, b=3 n^{1 / 3}$ 


\begin{tabular}{|c|c|c|c|c|c|c|c|c|c|c|c|c|c|}
\hline \multicolumn{2}{|c|}{$\overline{\mathrm{MAA}}(1), \delta=0.9$} & \multicolumn{4}{|c|}{$\begin{array}{l}n=100 \\
\end{array}$} & \multicolumn{4}{|c|}{$\begin{array}{l}n=400 \\
\end{array}$} & \multicolumn{4}{|c|}{$\bar{n} n=1200$} \\
\hline 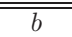 & & $\overline{\bar{\sigma} \sigma}$ & $\overline{\overline{m e a n}\left(\widehat{\sigma}^{*}\right)}$ & 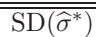 & $\overline{\mathrm{MSE}\left(\widehat{\sigma}^{*}\right)}$ & $\overline{\bar{\sigma}}$ & $\overline{\text { mean }\left(\widehat{\sigma}^{*}\right)}$ & 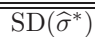 & $\overline{\mathrm{MSE}\left(\widehat{\sigma}^{*}\right)}$ & $\overline{\overline{\sigma \sigma}}$ & $\overline{\text { mean }\left(\widehat{\sigma}^{*}\right)}$ & $\overline{\mathrm{SSD}\left(\widehat{\sigma}^{*}\right)}$ & $\overline{\mathrm{MSE}\left(\widehat{\sigma}^{*}\right)}$ \\
\hline \multirow[t]{2}{*}{$\bar{c}^{n^{1 / 3}}$} & $\widehat{B_{11}}$ & 1.939 & 2.073 & 0.562 & 0.334 & 1.888 & 1.965 & 0.305 & 0.099 & 1.882 & 1.939 & 0.229 & 0.056 \\
\hline & $\widehat{B}_{12}$ & 2.178 & 2.388 & 0.644 & 0.458 & 2.192 & 2.264 & 0.354 & 0.130 & 2.182 & 2.237 & 0.264 & 0.073 \\
\hline \multirow[t]{2}{*}{$3 n^{1 / 3}$} & $\widehat{B}_{11}$ & 1.939 & 2.025 & 0.728 & 0.537 & 1.888 & 1.921 & 0.430 & 0.186 & 1.882 & 1.904 & 0.290 & 0.085 \\
\hline & $\widehat{B}_{12}$ & 2.178 & 2.327 & 0.859 & 0.760 & 2.192 & 2.239 & 0.497 & 0.249 & 2.182 & 2.212 & 0.339 & 0.116 \\
\hline \multirow[t]{2}{*}{$5 n^{1 / 3}$} & $\widehat{B}_{11}$ & 1.939 & 1.986 & 0.873 & 0.764 & 1.888 & 1.951 & 0.516 & 0.27 & 1.882 & 1.947 & 0.378 & 0.147 \\
\hline & $\widehat{B}_{12}$ & 2.178 & 2.306 & 0.843 & 0.726 & 2.192 & 2.292 & 0.607 & 0.379 & 2.182 & 2.237 & 0.415 & 0.175 \\
\hline
\end{tabular}

\begin{tabular}{|c|c|c|c|c|c|c|c|c|c|c|c|c|c|}
\hline \multicolumn{2}{|c|}{$\mathrm{MA}(1), \delta=0.5$} & \multicolumn{4}{|c|}{$n=100$} & \multicolumn{4}{|c|}{$n=400$} & \multicolumn{4}{|c|}{ "n=1200 } \\
\hline$\overline{\bar{b}}$ & & $\overline{\bar{\sigma} \sigma}$ & $\overline{\text { mean }\left(\widehat{\sigma}^{*}\right)}$ & 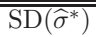 & $\overline{\mathrm{MSE}\left(\widehat{\sigma}^{*}\right)}$ & $\overline{\bar{\sigma}}$ & $\overline{\text { mean }\left(\widehat{\sigma}^{*}\right)}$ & 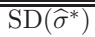 & 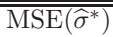 & $\overline{\bar{\sigma} \sigma}$ & $\overline{\text { mean }\left(\widehat{\sigma}^{*}\right)}$ & $\begin{array}{l}\mathrm{SD}\left(\widehat{\sigma}^{*}\right) \\
\end{array}$ & 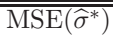 \\
\hline \multirow[t]{2}{*}{$n^{1 / 3}$} & $\widehat{\widehat{B_{11}}}$ & 2.421 & 2.476 & ב-642 & "0.415 & 2.371 & 2.486 & "0.387 & "0.163 & 2.394 & 2.417 & "0.270 & (20.073 \\
\hline & $\widehat{B}_{12}$ & 2.194 & 2.273 & 0.581 & 0.343 & 2.168 & 2.241 & 0.368 & 0.141 & 2.124 & 2.173 & 0.239 & 0.060 \\
\hline \multirow[t]{2}{*}{$3 n^{1 / 3}$} & $\widehat{B_{11}}$ & 2.421 & 2.526 & 0.907 & 0.834 & 2.371 & 2.436 & 0.512 & 0.267 & 2.394 & 2.392 & 0.345 & 0.119 \\
\hline & $\widehat{B}_{12}$ & 2.194 & 2.272 & 0.853 & 0.734 & 2.168 & 2.179 & 0.464 & 0.215 & 2.124 & 2.167 & 0.325 & 0.107 \\
\hline \multirow[t]{2}{*}{$5 n^{1 / 3}$} & $\widehat{B_{11}}$ & 2.421 & 2.443 & 1.066 & 1.136 & 2.371 & 2.489 & 0.677 & 0.472 & 2.394 & 2.428 & 0.451 & 0.205 \\
\hline & $\widehat{B}_{12}$ & 2.194 & 2.286 & 0.940 & 0.892 & 2.168 & 2.242 & 0.607 & 0.374 & 2.124 & 2.188 & 0.396 & 0.161 \\
\hline
\end{tabular}

\begin{tabular}{|c|c|c|c|c|c|c|c|c|c|c|c|c|c|}
\hline \multicolumn{2}{|c|}{ MA(1), $\delta=0.1$} & \multicolumn{4}{|c|}{$n=100$} & \multicolumn{4}{|c|}{$n=400$} & \multicolumn{4}{|c|}{$n=1200$} \\
\hline $\bar{b}$ & & $\bar{\sigma}$ & $\overline{\operatorname{mean}\left(\widehat{\sigma}^{*}\right)}$ & $\begin{array}{l}\mathrm{SD}\left(\widehat{\sigma}^{*}\right) \\
\end{array}$ & $\overline{\mathrm{MSE}\left(\widehat{\sigma}^{*}\right)}$ & $\bar{\sigma}$ & $\overline{\text { mean }\left(\widehat{\sigma}^{*}\right)}$ & \begin{tabular}{|l|l|}
$\mathrm{SD}\left(\hat{\sigma}^{*}\right)$ \\
\end{tabular} & $\overline{\mathrm{MSE}\left(\widehat{\sigma}^{*}\right)}$ & $\bar{\sigma}$ & $\overline{\text { mean }\left(\widehat{\sigma}^{*}\right)}$ & SD( $\left.\widehat{\sigma}^{*}\right)$ & $\overline{\mathrm{MSE}\left(\widehat{\sigma}^{*}\right)}$ \\
\hline \multirow[t]{2}{*}{$n^{1 / 3}$} & 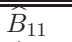 & (3.225 & 3.200 & 0.822 & "0.676 & 3.218 & (3.209 & 0.478 & 0.229 & (3.222 & ב3.235 & 0.365 & 0.134 \\
\hline & $\widehat{B}_{12}$ & 2.148 & 2.255 & 0.647 & 0.429 & 2.091 & 2.180 & 0.323 & 0.112 & 2.066 & 2.150 & 0.238 & 0.064 \\
\hline \multirow[t]{2}{*}{$3 n^{1 / 3}$} & $\widehat{B_{11}}$ & 3.225 & 3.199 & 1.155 & 1.335 & 3.218 & 3.280 & 0.675 & 0.46 & 3.222 & 3.275 & 0.507 & 0.260 \\
\hline & $\widehat{B}_{12}$ & 2.148 & 2.156 & 0.812 & 0.659 & 2.091 & 2.208 & 0.494 & 0.258 & 2.066 & 2.145 & 0.336 & 0.119 \\
\hline \multirow[t]{2}{*}{$5 n^{1 / 3}$} & $\widehat{B_{11}}$ & 3.225 & 3.224 & 1.263 & 1.595 & 3.218 & 3.314 & 0.827 & 0.694 & 3.222 & 3.331 & 0.611 & 0.385 \\
\hline & $\widehat{B}_{12}$ & 2.148 & 2.194 & 1.015 & 1.032 & 2.091 & 2.154 & 0.569 & 0.328 & 2.066 & 2.138 & 0.394 & 0.160 \\
\hline
\end{tabular}

TABLE 5. Standard deviations $\widehat{\sigma}^{*}$ of the entries of $n(\widehat{B}-B)$ estimated by the CBB bootstrap for the $\operatorname{VMA}(1)$ model a), sample sizes $n \in\{100,400,1200\}$, (rounded) block lengths $b \in\left\{n^{1 / 3}, 3 n^{1 / 3}, 5 n^{1 / 3}\right\}$ and $\delta \in\{0.9,0.5,0.1\}$. The true parameter $\sigma$ is estimated by 20,000 Monte Carlo replications.

for $\widehat{A}_{21}$ and $\widehat{A}_{11}$ and $b=5 n^{1 / 3}$ for $\widehat{A}_{12}$. Interestingly, if one compares the true (simulated) values of $\sigma$ depending on $\delta \in\{0.9,0.5,0.1\}$ in Tables $1-6$, it becomes apparent that in some cases a smaller parameter $\delta$ leads to larger $\sigma$, while this effect is reversed in other cases.

Finally, in Table 7, we show the bootstrap performance of the CBB in terms of coverage rates of bootstrap confidence intervals. In general, these turn out to be quite accurate and again the effect of the block length choice is not very pronounced.

\section{Conclusions}

In this paper, some asymptotic theory for block bootstrap resampling schemes has been developed when such procedures are applied to multivariate integrated and/or cointegrated time series. We proved a functional central limit theorem for the partial sum process based on pseudotime series generated using a multivariate continuous-path block bootstrap (CBB) procedure. The pseudo-time series generated in this context is based on integrating resampled blocks of the centered differences of the observed multivariate time series. This basic result is used to establish asymptotic validity of the CBB procedure for estimating the distribution of least squares estimators, both, in the full rank regression and in the spurious regression case. We showed further, that the CBB procedure fails in the case of cointegrated processes since it does not capture appropriately the cointegration structure of the underlying time series. For this kind 


\begin{tabular}{|c|c|c|c|c|c|c|c|c|c|c|c|c|c|}
\hline \multicolumn{2}{|c|}{$\overline{A R R}(1), \delta=0.9$} & \multicolumn{4}{|c|}{$n=100$} & \multicolumn{4}{|c|}{$n=400$} & \multicolumn{4}{|c|}{$\begin{array}{l}n=1200 \\
\end{array}$} \\
\hline 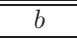 & & 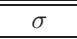 & "mean $\left(\widehat{\sigma}^{*}\right)$ & $\overline{\mathrm{SD}\left(\hat{\sigma}^{*}\right)}$ & $\overline{\mathrm{MSE}\left(\hat{\sigma}^{*}\right)}$ & $\bar{\sigma} \sigma$ & $\overline{\mathrm{m} e a n}\left(\widehat{\sigma}^{*}\right)$ & $\overline{\mathrm{SD}\left(\hat{\sigma}^{*}\right)}$ & $\overline{\mathrm{MSE}\left(\hat{\sigma}^{*}\right)}$ & $\overline{\sigma \sigma}$ & $\overline{\mathrm{m} e a n}\left(\widehat{\sigma}^{*}\right)$ & 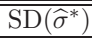 & $\operatorname{MSE}\left(\widehat{\sigma}^{*}\right)$ \\
\hline \multirow[t]{2}{*}{$n^{1 / 3}$} & $\widehat{B_{11}}$ & 0.519 & 0.894 & 0.309 & 0.236 & 0.394 & 0.666 & 0.132 & 0.091 & 0.371 & 0.536 & 0.066 & 0.032 \\
\hline & $\widehat{B}_{12}$ & 1.774 & 1.922 & 0.540 & 0.314 & 1.672 & 1.743 & 0.279 & 0.083 & 1.649 & 1.667 & 0.182 & 0.034 \\
\hline \multirow[t]{2}{*}{$3 n^{1 / 3}$} & $\widehat{B_{11}}$ & 0.519 & 0.668 & 0.300 & 0.112 & 0.394 & 0.477 & 0.125 & 0.023 & 0.371 & 0.420 & 0.071 & 0.007 \\
\hline & $\widehat{B}_{12}$ & 1.774 & 1.800 & 0.672 & 0.452 & 1.672 & 1.679 & 0.370 & 0.137 & 1.649 & 1.649 & 0.245 & 0.060 \\
\hline \multirow[t]{2}{*}{$5 n^{1 / 3}$} & $\widehat{B_{11}}$ & 0.519 & 0.655 & 0.366 & 0.153 & 0.394 & 0.454 & 0.135 & 0.022 & 0.371 & 0.406 & 0.079 & 0.007 \\
\hline & $\widehat{B}_{12}$ & 1.774 & 1.922 & 0.915 & 0.859 & 1.672 & 1.688 & 0.462 & 0.214 & 1.649 & 1.692 & 0.308 & 0.097 \\
\hline
\end{tabular}

\begin{tabular}{|c|c|c|c|c|c|c|c|c|c|c|c|c|c|}
\hline \multicolumn{2}{|c|}{$\overline{\mathrm{ARR}(1), \delta=0.5}$} & \multicolumn{4}{|c|}{$n=100$} & \multicolumn{4}{|c|}{$\bar{n} n=400$} & \multicolumn{4}{|c|}{$=1200$} \\
\hline $\bar{b}$ & & $\overline{\sigma \sigma}$ & "mean $\left(\widehat{\sigma}^{*}\right)$ & $\overline{\mathrm{SDD}\left(\widehat{\sigma}^{*}\right)}$ & 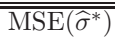 & $\overline{\sigma \sigma}$ & $\overline{\text { mean }\left(\widehat{\sigma}^{*}\right)}$ & $\overline{\mathrm{SD}\left(\widehat{\sigma}^{*}\right)}$ & 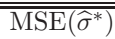 & $\overline{\sigma \sigma}$ & 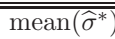 & $\overline{\mathrm{SD}\left(\widehat{\sigma}^{*}\right)}$ & $\overline{\mathrm{MSE}\left(\widehat{\sigma}^{*}\right)}$ \\
\hline \multirow[t]{2}{*}{$n^{1 / 3}$} & $\widehat{B_{11}}$ & 1.807 & 1.947 & 0.511 & 0.281 & 1.812 & 1.866 & 0.314 & 0.101 & 1.778 & 1.834 & 0.203 & 0.044 \\
\hline & $\widehat{B}_{12}$ & 1.716 & 1.885 & 0.500 & 0.278 & 1.648 & 1.774 & 0.274 & 0.091 & 1.656 & 1.716 & 0.182 & 0.037 \\
\hline \multirow[t]{2}{*}{$3 n^{1 / 3}$} & $\widehat{B_{11}}$ & 1.807 & 1.919 & 0.746 & 0.569 & 1.812 & 1.843 & 0.394 & 0.156 & 1.778 & 1.818 & 0.267 & 0.073 \\
\hline & $\widehat{B}_{12}$ & 1.716 & 1.805 & 0.693 & 0.488 & 1.648 & 1.730 & 0.411 & 0.176 & 1.656 & 1.670 & 0.267 & 0.072 \\
\hline \multirow[t]{2}{*}{$5 n^{1 / 3}$} & $\widehat{B_{11}}$ & 1.807 & 1.867 & 0.794 & 0.634 & 1.812 & 1.817 & 0.475 & 0.225 & 1.778 & 1.836 & 0.342 & 0.121 \\
\hline & $\widehat{B}_{12}$ & 1.716 & 1.710 & 0.704 & 0.496 & 1.648 & 1.703 & 0.479 & 0.232 & 1.656 & 1.699 & 0.310 & 0.098 \\
\hline
\end{tabular}

\begin{tabular}{|c|c|c|c|c|c|c|c|c|c|c|c|c|c|}
\hline \multicolumn{2}{|c|}{$\overline{\mathrm{AR}}(1), \delta=0.1$} & \multicolumn{4}{|c|}{$\bar{c} n=100$} & \multicolumn{4}{|c|}{$\bar{c} n=400$} & \multicolumn{4}{|c|}{$=n=1200$} \\
\hline 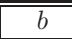 & & 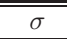 & mean $\left(\widehat{\sigma}^{*}\right)$ & $\overline{\mathrm{SD}\left(\widehat{\sigma}^{*}\right)}$ & $\overline{\mathrm{MSSE}\left(\widehat{\sigma}^{*}\right)}$ & $\overline{\bar{\sigma} \sigma}$ & 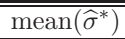 & $\overline{\mathrm{SDD}\left(\widehat{\sigma}^{*}\right)}$ & $\overline{\mathrm{MSSE}\left(\widehat{\sigma}^{*}\right)}$ & 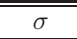 & $\overline{\mathrm{c} \operatorname{mean}\left(\widehat{\sigma}^{*}\right)}$ & $\overline{\mathrm{SD}\left(\widehat{\sigma}^{*}\right)}$ & $\overline{\mathrm{MSE}\left(\widehat{\sigma}^{*}\right)}$ \\
\hline \multirow[t]{2}{*}{$n^{1 / 3}$} & $\widehat{B_{11}}$ & 3.101 & 2.825 & 0.705 & 0.574 & 3.199 & 2.987 & 0.440 & 0.239 & 3.207 & 3.042 & 0.321 & 0.131 \\
\hline & $\widehat{B}_{12}$ & 1.661 & 1.872 & 0.516 & 0.311 & 1.656 & 1.788 & 0.285 & 0.099 & 1.631 & 1.707 & 0.191 & 0.042 \\
\hline \multirow[t]{2}{*}{$3 n^{1 / 3}$} & $\widehat{B_{11}}$ & 3.101 & 3.066 & 1.093 & 1.196 & 3.199 & 3.237 & 0.716 & 0.514 & 3.207 & 3.192 & 0.486 & 0.237 \\
\hline & $\widehat{B}_{12}$ & 1.661 & 1.778 & 0.684 & 0.481 & 1.656 & 1.749 & 0.380 & 0.153 & 1.631 & 1.687 & 0.260 & 0.071 \\
\hline \multirow[t]{2}{*}{$5 n^{1 / 3}$} & $\widehat{B_{11}}$ & 3.101 & 3.218 & 1.279 & 1.649 & 3.199 & 3.239 & 0.809 & 0.657 & 3.207 & 3.232 & 0.577 & 0.333 \\
\hline & $\widehat{B}_{12}$ & 1.661 & 1.840 & 0.794 & 0.662 & 1.656 & 1.708 & 0.433 & 0.191 & 1.631 & 1.685 & 0.308 & 0.098 \\
\hline
\end{tabular}

TABLE 6. Standard deviations $\widehat{\sigma}^{*}$ of the entries of $n(\widehat{B}-B)$ estimated by the CBB bootstrap for the $\operatorname{VAR}(1)$ model b), sample sizes $n \in\{100,400,1200\}$, (rounded) block lengths $b \in\left\{n^{1 / 3}, 3 n^{1 / 3}, 5 n^{1 / 3}\right\}$ and $\delta \in\{0.9,0.5,0.1\}$. The true parameter $\sigma$ is estimated by 20,000 Monte Carlo replications.

of integrated multivariate processes, a block resampling scheme based on regression residuals (RBB) is more appropriate. It is shown that the RBB procedure is valid for estimating the distribution of the least square estimator of the cointegration matrix.

\section{Proofs}

Proof of Theorem 2.1: First, note that

$$
\sum_{j=1}^{t} U_{t}^{*}=\sum_{m=0}^{M_{t}} \sum_{s=1}^{B_{t, m}} U_{m b+s}^{*}=\sum_{m=0}^{M_{t}} \sum_{s=1}^{B_{t, m}} \widehat{U}_{i_{m}+s},
$$

where $M_{t}=\left\lceil\frac{t}{b}\right\rceil-1, B_{t, m}=\min (b, t-m b)$ and

$$
\widehat{U}_{i_{m}+s}:=U_{i_{m}+s}-E^{*}\left(U_{i_{m}+s}\right)=U_{i_{m}+s}-\frac{1}{n-b+1} \sum_{\tau=0}^{n-b} U_{\tau+s} .
$$

Here, $\lceil x\rceil(\lfloor x\rfloor)$ denotes the smallest (largest) integer that is larger (smaller) or equal to $x$. Similarly, we set $M_{\nu}=\left\lfloor\frac{\lfloor l \nu\rfloor-1}{b}\right\rfloor$ and $B_{\nu, m}=\min (b,\lfloor l \nu\rfloor-m b)$ and together with the definition 


\begin{tabular}{||c||c||c|c|c||c|c|c||}
\hline \hline \multicolumn{1}{||c||}{$\delta=0.9$} & \multicolumn{2}{c||}{ VMA(1) model } & \multicolumn{3}{c||}{ VAR(1) model } \\
\hline \hline $\mathrm{b}$ & $\mathrm{n}$ & 100 & 400 & 1200 & 100 & 400 & 1200 \\
\hline \hline$n^{1 / 3}$ & $B_{11}$ & 0.936 & 0.952 & 0.940 & 0.990 & 0.990 & 0.986 \\
& $B_{12}$ & 0.928 & 0.958 & 0.950 & 0.948 & 0.938 & 0.958 \\
\hline $3 n^{1 / 3}$ & $B_{11}$ & 0.884 & 0.926 & 0.938 & 0.946 & 0.962 & 0.958 \\
& $B_{12}$ & 0.890 & 0.958 & 0.948 & 0.866 & 0.932 & 0.930 \\
\hline $5 n^{1 / 3}$ & $B_{11}$ & 0.816 & 0.934 & 0.952 & 0.896 & 0.934 & 0.956 \\
& $B_{12}$ & 0.828 & 0.926 & 0.914 & 0.844 & 0.926 & 0.920 \\
\hline \hline
\end{tabular}

\begin{tabular}{||c||c||c|c|c||c|c|c||}
\hline \hline \multicolumn{2}{||c||}{$\delta=0.5$} & \multicolumn{2}{c||}{ VMA(1) model } & \multicolumn{2}{c||}{ VAR(1) model } \\
\hline \hline $\mathrm{b}$ & $\mathrm{n}$ & 100 & 400 & 1200 & 100 & 400 & 1200 \\
\hline \hline$n^{1 / 3}$ & $B_{11}$ & 0.932 & 0.944 & 0.946 & 0.952 & 0.962 & 0.946 \\
& $B_{12}$ & 0.960 & 0.948 & 0.95 & 0.964 & 0.954 & 0.958 \\
\hline $3 n^{1 / 3}$ & $B_{11}$ & 0.916 & 0.930 & 0.946 & 0.890 & 0.920 & 0.950 \\
& $B_{12}$ & 0.910 & 0.910 & 0.942 & 0.850 & 0.908 & 0.938 \\
\hline $5 n^{1 / 3}$ & $B_{11}$ & 0.822 & 0.914 & 0.924 & 0.862 & 0.902 & 0.938 \\
& $B_{12}$ & 0.848 & 0.922 & 0.944 & 0.836 & 0.930 & 0.938 \\
\hline \hline
\end{tabular}

\begin{tabular}{||c||c||c|c|c||c|c|c||}
\hline \hline \multicolumn{1}{||c||}{$\delta=0.1$} & \multicolumn{2}{c||}{ VMA(1) model } & \multicolumn{3}{c||}{ VAR(1) model } \\
\hline \hline $\mathrm{b}$ & $\mathrm{n}$ & 100 & 400 & 1200 & 100 & 400 & 1200 \\
\hline \hline$n^{1 / 3}$ & $B_{11}$ & 0.922 & 0.918 & 0.938 & 0.922 & 0.924 & 0.930 \\
& $B_{12}$ & 0.950 & 0.938 & 0.946 & 0.958 & 0.936 & 0.926 \\
\hline $3 n^{1 / 3}$ & $B_{11}$ & 0.874 & 0.934 & 0.952 & 0.876 & 0.912 & 0.940 \\
& $B_{12}$ & 0.92 & 0.932 & 0.948 & 0.870 & 0.934 & 0.934 \\
\hline $5 n^{1 / 3}$ & $B_{11}$ & 0.854 & 0.928 & 0.928 & 0.824 & 0.914 & 0.932 \\
& $B_{12}$ & 0.840 & 0.924 & 0.928 & 0.810 & 0.934 & 0.942 \\
\hline \hline
\end{tabular}

TABLE 7. Coverage rates of CBB bootstrap confidence intervals for the entries of $B$ for the $\operatorname{VMA}(1)$ model a) and the $\operatorname{VAR}(1)$ model b), sample sizes $n \in$ $\{100,400,1200\}$, (rounded) block lengths $b \in\left\{n^{1 / 3}, 3 n^{1 / 3}, 5 n^{1 / 3}\right\}$ and $\delta \in$ $\{0.9,0.5,0.1\}$.

of $S_{l}^{*}(\nu)$ in $(2.5)$, this leads to

$$
\begin{aligned}
S_{l}^{*}(\nu) & =\frac{1}{\sqrt{l}}\left(\Omega_{l}^{*}\right)^{-1 / 2} \sum_{m=0}^{M_{\nu}} \sum_{s=1}^{B_{\nu, m}} \widehat{U}_{i_{m}+s} \\
& =\frac{1}{\sqrt{l}}\left(\Omega_{l}^{*}\right)^{-1 / 2} \sum_{m=0}^{M_{\nu}} \sum_{s=1}^{b} \widehat{U}_{i_{m}+s}-\frac{1}{\sqrt{l}}\left(\Omega_{l}^{*}\right)^{-1 / 2} \sum_{s=B_{\nu, M_{\nu}+1}}^{b} \widehat{U}_{i_{M_{\nu}}+s}
\end{aligned}
$$

and since

$$
\sup _{\nu}\left\|\frac{1}{\sqrt{l}}\left(\Omega_{l}^{*}\right)^{-1 / 2} \sum_{s=B_{\nu, M_{\nu}}+1}^{b} \widehat{U}_{i_{M_{\nu}}+s}\right\|=O_{P}\left(k^{-1 / 2}\right),
$$

it remains to show

$$
\left(\frac{1}{\sqrt{l}}\left(\Omega_{l}^{*}\right)^{-1 / 2} \sum_{m=0}^{M_{\nu}} \sum_{s=1}^{b} \widehat{U}_{i_{m}+s}\right)_{\nu \in[0,1]} \Rightarrow(W(\nu), \nu \in[0,1])
$$

in probability. We can consider instead the asymptotically equivalent statistic

$$
\widetilde{S}_{l}^{*}(\nu)=\frac{1}{\sqrt{l}}\left(\Omega_{l}^{*}\right)^{-1 / 2} \sum_{m=0}^{\lfloor k \nu\rfloor} \sum_{s=1}^{b} \widehat{U}_{i_{m}+s}=\frac{1}{\sqrt{k}} \sum_{m=0}^{\lfloor k \nu\rfloor} V_{m}^{*},
$$


where $\left\{V_{m}^{*}, m=0,1,2, \ldots,\lfloor k \nu\rfloor\right\}$ with

$$
V_{m}^{*}=\frac{1}{\sqrt{b}}\left(\Omega_{l}^{*}\right)^{-1 / 2} \sum_{s=1}^{b} \widehat{U}_{i_{m}+s}
$$

forms a triangular array of random variables that are independently and identically distributed conditionally on $X_{1}, \ldots, X_{n}$. Now, to prove $\widetilde{S}_{l}^{*} \Rightarrow W$, we have to show

(a) Convergence of all finite dimensional distributions

(b) Tightness

For the first part (a), let $q \in \mathbb{N}$ and set $\underline{\nu}=\left(\nu_{1}, \ldots, \nu_{q}\right)^{\prime}$, where $0 \leq \nu_{1} \leq \ldots \leq \nu_{q} \leq 1$. Further, let $\widetilde{S}_{l}^{*}(\underline{\nu})$ be the vector that stacks $\widetilde{S}_{l}^{*}\left(\nu_{1}\right), \ldots, \widetilde{S}_{l}^{*}\left(\nu_{q}\right)$ and $\Delta \widetilde{S}_{l}^{*}(\underline{\nu})$ be the vector that stacks the differences $\widetilde{S}_{l}^{*}\left(\nu_{1}\right)-\widetilde{S}_{l}^{*}\left(\nu_{0}\right), \ldots, \widetilde{S}_{l}^{*}\left(\nu_{q}\right)-\widetilde{S}_{l}^{*}\left(\nu_{q-1}\right)$, where we set $\nu_{0}=0$. Note that the $q m$ dimensional process $\left\{\Delta \widetilde{S}_{l}^{*}(\underline{\nu}), \underline{\nu} \in[0,1]^{q}\right\}$ consists of $q$ conditionally independent $m$-dimensional processes due to the independence of $\left\{V_{m}^{*}, m=0,1,2, \ldots,\lfloor k \nu\rfloor\right\}$. This allows us to consider them separately. We have

$$
\widetilde{S}_{l}^{*}\left(\nu_{p}\right)-\widetilde{S}_{l}^{*}\left(\nu_{p-1}\right)=\frac{1}{\sqrt{k}} \sum_{m=\left\lfloor k \nu_{p-1}\right\rfloor+1}^{\left\lfloor k \nu_{p}\right\rfloor} V_{m}^{*}=\frac{1}{\sqrt{l}}\left(\Sigma_{l}^{*}\right)^{-1 / 2} \sum_{m=\left\lfloor k \nu_{p-1}\right\rfloor+1}^{\left\lfloor k \nu_{p}\right\rfloor} \sum_{s=1}^{b} \widehat{U}_{i_{m}+s},
$$

and due to $E^{*}\left(V_{m}^{*}\right)=0$ and Lemma $6.1(\mathrm{i})$, we obtain

$$
\begin{aligned}
& \operatorname{Var}^{*}\left(\widetilde{S}_{l}^{*}\left(\nu_{p}\right)-\widetilde{S}_{l}^{*}\left(\nu_{p-1}\right)\right) \\
= & \frac{1}{k}\left\{\left(\Omega_{l}^{*}\right)^{-1 / 2} E^{*}\left[\sum_{m_{1}=\left\lfloor k \nu_{p-1}\right\rfloor+1}^{\left\lfloor k \nu_{p}\right\rfloor} \sum_{s_{1}=1}^{b} \widehat{U}_{i_{m_{1}}+s_{1}} \sum_{m_{2}=\left\lfloor k \nu_{p-1}\right\rfloor+1}^{\left\lfloor k \nu_{p}\right\rfloor} \sum_{s_{2}=1}^{b} \widehat{U}_{i_{m_{2}}+s_{2}}^{\prime}\right]\left(\Omega_{l}^{*}\right)^{-1 / 2}\right\} \\
= & \frac{\left\lfloor k \nu_{p}\right\rfloor-\left\lfloor k \nu_{p-1}\right\rfloor}{k}\left\{\left(\Omega_{l}^{*}\right)^{-1 / 2}\left(\Omega_{\left\lfloor k \nu_{p}\right\rfloor-\left\lfloor k \nu_{p-1}\right\rfloor}^{*}\right)\left(\Omega_{l}^{*}\right)^{-1 / 2}\right\} \\
\substack{k \rightarrow \infty \\
\rightarrow \infty} & \left(\nu_{p}-\nu_{p-1}\right) I_{m}
\end{aligned}
$$

as $n \rightarrow \infty$ in probability for any $p$. To prove asymptotic normality, we show a CLT based on the Liapunov condition for triangular arrays $\left\{V_{m}^{*}, m=0,1,2, \ldots,\lfloor k \nu\rfloor\right\}$ [see e.g. Serfling (1980)]. More precisely and due to $k^{-1} \operatorname{Var}^{*}\left(\sum_{m=\left\lfloor k \nu_{p-1}\right\rfloor+1}^{\left\lfloor k \nu_{p}\right\rfloor} V_{m}^{*}\right)=O_{P}(1)$, it suffices to show

$$
\left(\frac{1}{\sqrt{k}}\right)^{2+\kappa} \sum_{m=\left\lfloor k \nu_{p-1}\right\rfloor+1}^{\left\lfloor k \nu_{p}\right\rfloor} E^{*}\left(\left\|V_{m}^{*}\right\|^{2+\kappa}\right)=o_{P}(1) \text {. }
$$

The latter results follows from

$$
\begin{aligned}
\frac{1}{k^{(2+\kappa) / 2}} \sum_{m=\left\lfloor k \nu_{p-1}\right\rfloor+1}^{\left\lfloor k \nu_{p}\right\rfloor} E^{*}\left(\left\|V_{m}^{*}\right\|^{2+\kappa}\right) & =\frac{\left(\left\lfloor k \nu_{p}\right\rfloor-\left\lfloor k \nu_{p-1}\right\rfloor\right)}{k^{(2+\kappa) / 2}} \frac{1}{n-b+1} \sum_{t=0}^{n-b}\left\|\frac{1}{\sqrt{b}}\left(\Omega_{l}^{*}\right)^{-1 / 2} \sum_{s=1}^{b} \widehat{U}_{t+s}\right\|^{2+\kappa} \\
& =O_{P}\left(\frac{1}{k^{\kappa / 2}}\right)
\end{aligned}
$$

because of

$\frac{1}{n-b+1} \sum_{t=0}^{n-b}\left\|\frac{1}{\sqrt{b}}\left(\Omega_{l}^{*}\right)^{-1 / 2} \sum_{s=1}^{b} \widehat{U}_{t+s}\right\|^{2+\kappa}=\int_{0}^{1}\left\|\widetilde{S}_{b}(\nu)\right\|^{2+\kappa} d \nu \Rightarrow \int_{0}^{1}\|W(\nu)\|^{2+\kappa} d \nu=O_{P}(1)$.

Here, the partial sum process $\left\{\widetilde{S}_{b}(\nu), 0 \leq \nu \leq 1\right\}$ is similarly defined as $\left\{S_{l}^{*}(\nu), 0 \leq \nu \leq 1\right\}$ in (2.5) and fulfills a FLT under the imposed conditions. Now, we can conclude that $S_{l}^{*}\left(\nu_{p}\right)-$ 
$S_{l}^{*}\left(\nu_{p-1}\right) \Rightarrow W\left(\nu_{p}-\nu_{p-1}\right)$ in probability. Together, we have that $\Delta \widetilde{S}_{l}^{*}(\underline{\nu})$ converges weakly to $\left(W^{\prime}\left(\nu_{1}-\nu_{0}\right), \ldots, W^{\prime}\left(\nu_{q}-\nu_{q-1}\right)\right)^{\prime}$. Finally, multiplying $\Delta \widetilde{S}_{l}^{*}(\underline{\nu})$ with a suitable matrix gives $\widetilde{S}_{l}^{*}(\underline{\nu})$ and by $W(0)=0$ and $W(a-b)=W(a)-W(b)$ for $a, b \in[0,1]$, we get the desired convergence of the finite dimensional distributions. For the tightness of part (b) recall first that in probability measures on the product space are tight if and oly if the marginal probability measures are tight; cf. Billingsley (1999), page 65. Now, for $i \in\{1,2, \ldots, m\}$ consider

$$
\widetilde{S}_{i, l}^{*}(\nu)=\underline{e}_{i}^{\prime} \widetilde{S}_{l}^{*}(\nu),
$$

where $\underline{e}_{i}=(0, \ldots, 0,1,0, \ldots, 0)$ is the $i$-th unit vector. Denote by $P_{i, l}^{*}$ the probability measure of $\widetilde{S}_{i, l}^{*}$ and by $P_{l}^{*}$ the probability measure of $\widetilde{S}_{l}^{*}$. Then, by a version of Donsker's Theorem for triangular arrays of row-wise independent random variables, Billingsley (1999), pages 147-148, we immediately get that $P_{i, l}^{*}$ is tight from which the tightness of $P_{l}^{*}$ follows.

Proof of Theorem 2.2: It holds

$$
l\left(\widehat{A}^{*}-I_{m}\right)=\left(\frac{1}{l} \sum_{t=2}^{l} U_{t}^{*} X_{t-1}^{*^{\prime}}\right)\left(\frac{1}{l^{2}} \sum_{t=2}^{l} X_{t-1}^{*} X_{t-1}^{*^{\prime}}\right)^{-1}
$$

and the claimed result follows from Lemma 6.1 and the positive definiteness of $\Omega$.

Lemma 6.1. Under the assumptions of Theorem 2.2, it holds

$$
\begin{aligned}
& \text { (i) } \Omega_{l}^{*} \rightarrow \Omega \quad \text { in probability, } \\
& \text { (ii) } \frac{1}{l} \sum_{t=2}^{l} U_{t}^{*} X_{t-1}^{*^{\prime}} \Rightarrow \Omega^{1 / 2} \int_{0}^{1} W(t) d W^{\prime}(t) \Omega^{1 / 2}+\Omega_{1}, \\
& \text { (iii) } \frac{1}{l^{2}} \sum_{t=2}^{l} X_{t-1}^{*} X_{t-1}^{*^{\prime}} \Rightarrow \Omega^{1 / 2} \int_{0}^{1} W(t) W(t)^{\prime} d t \Omega^{1 / 2}
\end{aligned}
$$

in probability, respectively, where joint convergence (of (ii) and (iii)) also applies.

Proof: (i) By (2.6) and rewriting the involved sums, we get

$$
\begin{aligned}
\Omega_{l}^{*} & =\operatorname{Var}^{*}\left(\frac{1}{\sqrt{l}} \sum_{t=1}^{l} U_{t}^{*}\right) \\
& =\frac{1}{b} \sum_{s_{1}, s_{2}=1}^{b} \frac{1}{n-b+1} \sum_{t=0}^{n-b} \widehat{U}_{t+s_{1}} \widehat{U}_{t+s_{2}}^{\prime} \\
& =\sum_{h=-b-1}^{b-1} \frac{1}{b} \sum_{r=\max (1,1-h)}^{\min (b, b-h)} \frac{1}{n-b+1} \sum_{t=0}^{n-b} \widehat{U}_{t+r+h} \widehat{U}_{t+r}^{\prime} \\
& =\sum_{h=-(b-1)}^{b-1}\left\{\frac{1}{n-b+1} \sum_{t=1}^{n} \frac{b-|h|}{b} \widehat{U}_{t+h} \widehat{U}_{t}^{\prime}\right\}+O\left(\frac{b^{2}}{n}\right) \\
& =A_{q}+\left(A-A_{q}\right),
\end{aligned}
$$

where $A$ denotes the first term in (6.3) and $A_{q}$ the same sum with $\sum_{h=-(b-1)}^{b-1}$ replaced by $\sum_{h=-(q-1)}^{q-1}$ for some fixed $q$. Now, we use Proposition 6.3.9 in Brockwell and Davis (1991). Under the assumptions, we have for any fixed $h \in \mathbb{Z}$ that $\frac{1}{n-b+1} \sum_{t=1}^{n} \frac{b-|h|}{b} \widehat{U}_{t+h} \widehat{U}_{t}^{\prime} \rightarrow \Gamma(h)$ holds 
in probability as $n \rightarrow \infty$ and we get

$$
\sum_{h=-(q-1)}^{q-1}\left\{\frac{1}{n-b+1} \sum_{t=1}^{n} \frac{b-|h|}{b} \widehat{U}_{t+h} \widehat{U}_{t}^{\prime}\right\}+O\left(\frac{b^{2}}{n}\right) \underset{n \rightarrow \infty}{\rightarrow} \sum_{h=-q}^{q} \Gamma(h) \underset{q \rightarrow \infty}{\rightarrow} \sum_{h=-\infty}^{\infty} \Gamma(h)
$$

in probability. It remains to show for all $\delta>0$ that $\lim _{q \rightarrow \infty} \lim \sup _{n \rightarrow \infty} P^{*}\left(\left\|A-A_{q}\right\| \geq \delta\right)=0$ in probability, which can be proved by standard arguments.

(ii) Plugging-in for $X_{t-1}^{* \prime}$ leads to

$$
\begin{aligned}
\frac{1}{l} \sum_{t=2}^{l} U_{t}^{*} X_{t-1}^{* \prime} & =\frac{1}{l} \sum_{t=1}^{l}\left\{U_{t}^{*} \sum_{j=1}^{t-1} U_{j}^{* \prime}\right\}+\left(\frac{1}{l} \sum_{t=1}^{l} U_{t}^{*}\right) X_{0}^{\prime}-\frac{1}{l} U_{1}^{*} X_{0} \\
& =\frac{1}{l} \sum_{p=0}^{k-1} \sum_{r=1}^{b}\left\{U_{p b+r}^{*}\left(\sum_{m=0}^{M_{p b+r-1} B_{p b+r-1, m}} \sum_{s=1}^{*} U_{m b+s}^{* \prime}\right)\right\}+O_{P^{*}}\left(\frac{1}{\sqrt{l}}\right) \\
& =\frac{1}{l} \sum_{p=0}^{k-1} \sum_{r=1}^{b}\left\{\widehat{U}_{i_{p}+r}\left(\sum_{m=0}^{p-1} \sum_{s=1}^{b} \widehat{U}_{i_{m}+s}^{\prime}\right)\right\}+\frac{1}{l} \sum_{p=0}^{k-1} \sum_{r=1}^{b}\left\{\widehat{U}_{i_{p}+r}\left(\sum_{s=1}^{r-1} \widehat{U}_{i_{p}+s}\right)\right\}+O_{P^{*}}\left(\frac{1}{\sqrt{l}}\right) \\
& =A_{1}^{*}+A_{2}^{*}+O_{P^{*}}\left(\frac{1}{\sqrt{l}}\right) .
\end{aligned}
$$

with an obvious notation for $A_{1}^{*}$ and $A_{2}^{*}$. The first term can be expressed as

$$
A_{1}^{*}=\left(\Omega_{l}^{*}\right)^{1 / 2}\left\{\frac{1}{k} \sum_{p=0}^{k-1} V_{p}^{*}\left(\sum_{m=0}^{p-1} V_{m}^{*}\right)^{\prime}\right\}\left(\Omega_{l}^{*}\right)^{1 / 2}
$$

where $V_{m}^{*}$ is defined in (6.2) which forms a triangular array of random variables that are independently distributed conditionally on $X_{1}, \ldots, X_{n}$. By adopting the proof of Theorem 2.4(ii) in Chan and Wei (1988) for our purposes and the FLT in Theorem 2.1, we can show that

$$
\frac{1}{k} \sum_{p=0}^{k-1} V_{p}^{*}\left(\sum_{m=0}^{p-1} V_{m}^{*}\right)^{\prime} \Rightarrow \int_{0}^{1} W(t) d W^{\prime}(t)
$$

in probability. This is possible, because their proof is based exclusively on the FLT and, therefore, the fact that $\left\{V_{m}^{*}\right\}$ is a triangular array does not alter the proof. For $A_{2}^{*}$, we get

$$
E^{*}\left(A_{2}^{*}\right)=\frac{1}{l} \sum_{p=0}^{k-1} \sum_{r=1}^{b} \sum_{s=1}^{r-1} E^{*}\left(\widehat{U}_{i_{p}+r} \widehat{U}_{i_{p}+s}^{\prime}\right)=\frac{1}{b} \sum_{r=1}^{b} \sum_{s=1}^{r-1} \frac{1}{n-b+1} \sum_{t=0}^{n-b} \widehat{U}_{t+r} \widehat{U}_{t+s}^{\prime}
$$

which converges to $\Omega_{1}$ by Proposition 6.3.9 in Brockwell and Davis (1991). Similar arguments show $\operatorname{Var}^{*}\left(A_{2}^{*}\right)=o_{P}(1)$. 
(iii) Since $X_{t}^{*}=X_{0}+\sum_{j=1}^{t} Y_{j}^{*}=X_{0}+\sum_{j=1}^{t} U_{j}^{*}$, we get

$$
\begin{aligned}
\frac{1}{l^{2}} \sum_{t=2}^{l} X_{t-1}^{*} X_{t-1}^{* \prime} & =\frac{1}{l} \sum_{t=2}^{l}\left(\frac{1}{\sqrt{l}} \sum_{j=1}^{t-1} U_{j}^{*}\right)\left(\frac{1}{\sqrt{l}} \sum_{j=1}^{t-1} U_{j}^{* \prime}\right)+O_{P^{*}}\left(\frac{1}{\sqrt{l}}\right) \\
& =\left(\Omega_{l}^{*}\right)^{1 / 2} \frac{1}{l} \sum_{t=2}^{l}\left(\frac{1}{\sqrt{l}}\left(\Omega_{l}^{*}\right)^{-1 / 2} \sum_{j=1}^{t-1} U_{j}^{*}\right)\left(\frac{1}{\sqrt{l}} \sum_{j=1}^{t-1} U_{j}^{* \prime}\left(\Omega_{l}^{*}\right)^{-1 / 2}\right)\left(\Omega_{l}^{*}\right)^{1 / 2}+O_{P^{*}}\left(\frac{1}{\sqrt{l}}\right) \\
& =\left(\Omega_{l}^{*}\right)^{1 / 2} \frac{1}{l} \sum_{t=2}^{l} S_{l}^{*}\left(\frac{t-1}{l}\right) S_{l}^{* \prime}\left(\frac{t-1}{l}\right)\left(\Omega_{l}^{*}\right)^{1 / 2}+O_{P^{*}}\left(\frac{1}{\sqrt{l}}\right) \\
& =\left(\Omega_{l}^{*}\right)^{1 / 2} \int_{0}^{1} S_{l}^{*}([l \nu]) S_{l}^{* \prime}([l \nu]) d \nu\left(\Omega_{l}^{*}\right)^{1 / 2}+O_{P^{*}}\left(\frac{1}{\sqrt{l}}\right) \\
& \Rightarrow \Omega^{1 / 2} \int_{0}^{1} W(\nu) W^{\prime}(\nu) d \nu \Omega^{1 / 2},
\end{aligned}
$$

in probability by Theorem 2.1, part (i) of this lemma and the continuous mapping theorem.

Proof of Theorem 2.3: Due to (2.13), the claimed result follows immediately from Lemma 6.2 below.

Lemma 6.2. Under the assumptions of Theorem 2.3, it holds

(i) $\quad l^{-3 / 2} \sum_{t=1}^{l} X_{t}^{*} \Rightarrow \Omega^{1 / 2} \int_{0}^{1} W(\nu) d \nu$

(ii) $l^{-2} \sum_{t=1}^{l} X_{t}^{*} X_{t}^{*^{\prime}} \Rightarrow \Omega^{1 / 2} \int_{0}^{1} W(\nu) W^{\prime}(\nu) d \nu \Omega^{1 / 2}$,

(iii) $l^{-2} \sum_{t=1}^{l}\left(X_{t}^{*}-\bar{X}^{*}\right)\left(X_{t}^{*}-\bar{X}^{*}\right)^{\prime} \Rightarrow \Omega^{1 / 2}\left\{\int_{0}^{1} W(t) W^{\prime}(t) d t-\int_{0}^{1} W(t) d t \int_{0}^{1} W^{\prime}(t) d t\right\} \Omega^{1 / 2}$.

Proof: Considering part (i), Theorem 2.1, Lemma 6.1 and the continuous mapping theorem, gives

$$
\begin{aligned}
l^{-3 / 2} \sum_{t=1}^{l} X_{t}^{*} & =l^{-3 / 2} \sum_{t=1}^{l} \sum_{j=1}^{t-1} U_{j}^{*}+l^{-1 / 2} X_{0}+l^{-3 / 2} \sum_{t=1}^{l} U_{t}^{*} \\
& =\left(\Omega_{l}^{*}\right)^{1 / 2} \frac{1}{l} \sum_{t=1}^{l} \frac{1}{\sqrt{l}}\left(\Omega_{l}^{*}\right)^{-1 / 2} \sum_{j=1}^{t-1} U_{j}^{*}+O_{P^{*}}\left(l^{-1 / 2}\right)+O_{P^{*}}\left(l^{-1}\right) \\
& =\left(\Omega_{l}^{*}\right)^{1 / 2} \frac{1}{l} \sum_{t=1}^{l} S_{l}^{*}\left(\frac{t-1}{l}\right)+O_{P^{*}}\left(l^{-1 / 2}\right) \\
& =\left(\Omega_{l}^{*}\right)^{1 / 2} \sum_{t=1}^{l} \int_{\frac{t-1}{l}}^{\frac{t}{l}} S_{l}^{*}(\lfloor l \nu\rfloor) d \nu+O_{P^{*}}\left(l^{-1 / 2}\right) \\
& =\left(\Omega_{l}^{*}\right)^{1 / 2} \int_{0}^{1} S_{l}^{*}(\lfloor l \nu\rfloor) d \nu+O_{P^{*}}\left(l^{-1 / 2}\right) \\
& \Rightarrow \Omega^{1 / 2} \int_{0}^{1} W(\nu) d \nu
\end{aligned}
$$


in probability. Part (ii) follows from $l^{-2} \sum_{t=1}^{l} X_{t}^{*} X_{t}^{*^{\prime}}=l^{-2} \sum_{t=2}^{l} X_{t-1}^{*} X_{t-1}^{*^{\prime}}+O_{P^{*}}\left(\frac{1}{l}\right)$ and Lemma 6.1 and (iii) is an immediate consequence of (i) and (ii) of this lemma, due to

$$
l^{-2} \sum_{t=1}^{l}\left(X_{t}^{*}-\bar{X}^{*}\right)\left(X_{t}^{*}-\bar{X}^{*}\right)^{\prime}=l^{-2} \sum_{t=1}^{l} X_{t}^{*} X_{t}^{*^{\prime}}-\left(l^{-3 / 2} \sum_{t=1}^{l} X_{t}^{*}\right)\left(l^{-3 / 2} \sum_{t=1}^{l} X_{t}^{*}\right)^{\prime} .
$$

Proof of Theorem 3.1: It holds

$$
l\left(\widehat{B}^{+}-\widehat{B}\right)=\left(\frac{1}{l} \sum_{t=1}^{l} U_{1 t}^{+} X_{2 t}^{+\prime}\right)\left(\frac{1}{l^{2}} \sum_{t=1}^{l} X_{2 t}^{+} X_{2 t}^{+\prime}\right)^{-1}
$$

and the claimed result follows from Lemma 6.3.

Lemma 6.3. Let $\Omega_{l}^{+}$be defined analogue to (2.6), but based on $X_{1}^{+}, \ldots, X_{n}^{+}$. Under the assumptions of Theorem 3.1, it holds

$$
\begin{aligned}
& \text { (i) } \Omega_{l}^{+} \rightarrow \Omega \quad \text { in probability, } \\
& \text { (ii) } \frac{1}{l} \sum_{t=1}^{l} U_{1 t}^{+} X_{2 t}^{+\prime} \Rightarrow\left[\Omega^{1 / 2} \int_{0}^{1} W(t) d W^{\prime}(t) \Omega^{1 / 2}+\Omega_{0}+\Omega_{1}\right]_{12}, \\
& \text { (iii) } \frac{1}{l^{2}} \sum_{t=1}^{l} X_{2 t}^{+} X_{2 t}^{+\prime} \Rightarrow\left[\Omega^{1 / 2} \int_{0}^{1} W(t) W^{\prime}(t) d t \Omega^{1 / 2}\right]_{22},
\end{aligned}
$$

in probability, respectively, where joint convergence (of (ii) and (iii)) also applies.

Proof: First, by (3.1) and (3.7), we get

$$
\begin{aligned}
\left(\begin{array}{c}
U_{1, m b+j}^{+} \\
U_{2, m b+j}^{+}
\end{array}\right)= & \left(\begin{array}{c}
\widetilde{U}_{1, i_{m}+j} \\
U_{2, i_{m}+j}
\end{array}\right)-\frac{1}{n-b+1} \sum_{\tau=0}^{n-b}\left(\begin{array}{c}
\widetilde{U}_{1, \tau+j} \\
U_{2, \tau+j}
\end{array}\right) \\
= & \left(\begin{array}{c}
U_{1, i_{m}+j} \\
U_{2, i_{m}+j}
\end{array}\right)-\frac{1}{n-b+1} \sum_{\tau=0}^{n-b}\left(\begin{array}{c}
U_{1, \tau+j} \\
U_{2, \tau+j}
\end{array}\right) \\
& -\left(\begin{array}{c}
(\widehat{B}-B) X_{2, i_{m}+j} \\
0
\end{array}\right)-\frac{1}{n-b+1} \sum_{\tau=0}^{n-b}\left(\begin{array}{c}
(\widehat{B}-B) X_{2, \tau+j} \\
0
\end{array}\right)
\end{aligned}
$$

for $j=1, \ldots, b$ and $m=0, \ldots, k-1$.

(ii) Plugging-in for $X_{t-1}^{+\prime}$ leads to

$$
\begin{aligned}
\frac{1}{l} \sum_{t=1}^{l} U_{1 t}^{+} X_{2 t}^{+\prime} & =\frac{1}{l} \sum_{t=1}^{l}\left\{U_{1 t}^{+} \sum_{j=1}^{t-1} U_{2 j}^{+\prime}\right\}+\frac{1}{l} \sum_{t=1}^{l} U_{1 t}^{+} U_{2 t}^{+\prime}+\left(\frac{1}{l} \sum_{t=1}^{l} U_{1 t}^{+}\right) X_{20}^{\prime} \\
& =\frac{1}{l} \sum_{t=1}^{l}\left\{U_{1 t}^{+} \sum_{j=1}^{t-1} U_{2 j}^{+\prime}\right\}+\frac{1}{l} \sum_{t=1}^{l} U_{1 t}^{+} U_{2 t}^{+\prime}+O_{P^{+}}\left(\frac{1}{\sqrt{l}}\right) .
\end{aligned}
$$

The asymptotic behavior of the first term above follows by using (6.4) immediately from Lemma 6.1(ii), where the second term on the last right-hand side of (6.4) vanishes asymptotically due 
to $(\widehat{B}-B)=O_{P}\left(n^{-1}\right)$. Similarly, for the second term, we have

$$
\begin{aligned}
E^{+}\left(\frac{1}{l} \sum_{t=1}^{l} U_{1 t}^{+} U_{2 t}^{+\prime}\right) & =\frac{1}{l} \sum_{m=0}^{k-1} \sum_{s=1}^{b} E^{+}\left(U_{1, i_{m}+s} U_{2, i_{m}+s}^{\prime}\right)+o_{P}(1) \\
& =\frac{1}{b} \sum_{s=1}^{b} \frac{1}{n-b+1} \sum_{\tau=0}^{n-b} U_{1, \tau+s} U_{2, \tau+s}^{\prime}+o_{P}(1),
\end{aligned}
$$

which converges to $\Omega_{0}$ by Proposition 6.3.9 in Brockwell and Davis (1991). Similar arguments show that its conditional variance vanishes asymptotically in probability. Part (i) follows by similar arguments from (6.4) and Lemma 6.1(i) and part (iii) follows immediately from Lemma 6.1(iii).

Acknowledgement 1. The research of the first author was supported by a fellowship within the PhD-Program of the German Academic Exchange Service (DAAD) when Carsten Jentsch was visiting the University of California, San Diego, USA and partly from the Research Center (SFB) 884 "Political Economy of Reforms" (Project B6), funded by the German Research Foundation (DFG). The research of Dimitris Politis was partially supported by NSF grants DMS 13-08319 and DMS 12-2313\%.

\section{REFERENCES}

[1] Badillo, R., Belaire-Franch, J. and Reverte, C. (2010). Residual-based block bootstrap for cointegration testing. Applied Economics Letters, 17, 999-1003.

[2] Billingsley, P. (1999). Convergence of Probability Measures. John Wiley \& Sohns, Inc.: New York.

[3] Brockwell, P. J. and Davis, R. A. (1991). Time series: theory and methods. 2nd ed. Springer-Verlag, Berlin.

[4] Cavaliere, G., Rahbek, A. and Taylor, A. M. R. (2010a). Co-integration rank testing under conditional heteroskedasticity. Econometric Theory, 26, 1719-1760.

[5] Cavaliere, G., Rahbek, A. and Taylor, A. M. R. (2010b). Testing for co-integration in vector autoregressions with non-stationary volatility. Journal of Econometrics, 2158, 7-24.

[6] Cavaliere, G., Rahbek, A. and Taylor, A. M. R. (2012). Bootstrap determination of the co-integration rank in VAR models. Econometrica, 80, 1721-1740.

[7] Cavaliere, G., Rahbek, A. and Taylor, A. M. R. (2014). Bootstrap determination of the co-integration rank in heteroskedastic VAR models. Econometric Reviews, 33, 606-650.

[8] Chan, N. H. and Wei, C. Z. (1988). Limiting Distributions of Least Squares Estimates of Unstable Autoregressive Processes. Annals of Statistics, 16, 367-401.

[9] Chang, Y., Park, J.Y. and Song, K. (2006). Bootstrapping cointegrating regressions. Journal of Econometrics, 133, 703-739.

[10] Künsch, H. R. (1989). The Jackknife and the Bootstrap for General Stationary Observations. Ann. Statist., 17, No. 3, 1217-1241.

[11] Lahiri, S. N. (2003). Resampling methods for dependent data. Springer Series in Statistics. New York, NY: Springer.

[12] Li, H. and Maddala, G. S. (1997). Bootstrapping Cointegrating Regressions. Journal of Econometrics, 80, $297-318$.

[13] Liu, R. Y. and Singh, K. (1992). Moving blocks jackknife and bootstrap capture weak dependence. In: Exploring the Limits of Bootstrap (Eds.: LePage, R. and Billard, L.), 225-248. Wiley, New York.

[14] Lütkepohl (2005). New Introduction to Multiple Time Series Analysis. Berlin: Springer.

[15] Hamilton, J. D. (1994). Time Series Analysis. Princeton University Press, Princeton, New Jersey.

[16] Omtzigt, P. and Fachin, S. (2006). The size and power of bootstrap and Bartlett-corrected tests of hypotheses on the cointegration vectors. Econometric Reviews, 25, 41-60.

[17] Paparoditis, E. and Politis, D. N. (2001) Unit root testing via the continuous-path block bootstrap, Discussion Paper 20012006, Department of Economics, University of California, San Diego, USA.

[18] Paparoditis, E. and Politis, D. N. (2003). Residual-based block bootstrap for unit root testing. Econometrica, 71, 813-855.

[19] Parker, C., Paparoditis, E. and Politis, D. N. (2006). Unit root testing via the stationary bootstrap. Journal of Econometrics, 133, 601-638.

[20] Phillips, P. C. B. (1986). Understanding spurious regressions in econometrics. Journal of Econometrics, 33, 311-340. 
[21] Phillips, P. C. B. (1988). Weak Convergence to the Matrix Stochastic Integral $\int_{0^{1}}$ BdB'. Journal of Mulitvariate Analysis, 24, 252-264.

[22] Phillips, P. C. B. (2001). Bootstrapping spurious regression. Cowles Foundation Discussion Paper \# 1331, Yale University.

[23] Phillips, P. C. B. (2010). Bootstrapping I(1) data. Journal of Econometrics, 158, 280-284.

[24] Phillips, P. C. B. and Durlauf, S. N. (1986). Multiple time series regression with integrated processes. Rev. Econ. Stud., 53, 473-495.

[25] Serfling, R. (1980). Approximation Theorems of Mathematical Statistics. New York: John Wiley.

[26] Swensen, A. R. (2006). Bootstrap algorithms for testing and determining the cointegration rank in VAR models. Econometrica, 74, 1699-1714.

[27] Swensen, A. R. (2009). Corrigendum to Bootstrap algorithms for testing and determining the cointegration rank in VAR models. Econometrica, 77, 1703-1704.

[28] Swensen, A. R. (2011). A bootstrap algorithm for testing cointegration rank in VAR models in the presence of stationary variables. Journal of Econometrics, 165, 152-162.

[29] Trenkler, C. (2009). Bootstrapping Systems Cointegration Tests With a Prior Adjustment for Deterministic Terms. Econometric Theory, 25, 243-269.

Department of Economics, University of Mannheim, L7, 3-5, 68131 Mannheim, Germany

E-mail address: cjentsch@mail.uni-mannheim.de

Department of Mathematics and Statistics, University of Cyprus, P.O.Box 20537, CY-1678 Nicosia, Cyprus

E-mail address: stathis@ucy.ac.cy

Department of Mathematics, University of California, San Diego, La Jolla, CA 92093-0112, USA

E-mail address: politis@math.ucsd.edu 